\title{
Performance Enhancement for Multihop Cognitive DF and AF Relaying Protocols under Joint Impact of Interference and Hardware Noises: NOMA for Primary Network and Best-Path Selection for Secondary Network
}

\author{
Tran Trung Duy $\mathbb{D}^{1},{ }^{1}$ Pham Thi Dan Ngoc $\mathbb{D}^{1,2}$ and Phuong T. Tran ${ }^{3}{ }^{3}$ \\ ${ }^{1}$ Posts and Telecommunications Institute of Technology (PTIT), Ho Chi Minh City, Vietnam \\ ${ }^{2}$ Ho Chi Minh City University of Technology, VNU-HCM, Ho Chi Minh City, Vietnam \\ ${ }^{3}$ Wireless Communications Research Group, Faculty of Electrical and Electronics Engineering, Ton Duc Thang University, \\ Ho Chi Minh City, Vietnam
}

Correspondence should be addressed to Phuong T. Tran; tranthanhphuong@tdtu.edu.vn

Received 16 March 2020; Revised 7 March 2021; Accepted 29 March 2021; Published 13 April 2021

Academic Editor: Michael McGuire

Copyright (c) 2021 Tran Trung Duy et al. This is an open access article distributed under the Creative Commons Attribution License, which permits unrestricted use, distribution, and reproduction in any medium, provided the original work is properly cited.

\begin{abstract}
In this paper, we propose and evaluate performance of multihop multipath underlay cognitive radio networks. In a primary network, an uplink nonorthogonal multiple access method is employed to allow primary transmitters to simultaneously transmit their data to a primary receiver. Using an underlay spectrum-sharing method, secondary source and secondary relays must adjust their transmit power to guarantee quality of service of the primary network. Under the limited transmit power, cochannel interference from the primary transmitters, and hardware noises caused by impairments, we propose best-path selection methods to improve the end-to-end performance for the secondary network. Moreover, both multihop decode-andforward and amplify-and-forward relaying protocols are considered in this paper. We derive expressions of outage probability for the primary and secondary networks and propose an efficient method to calculate the transmit power of the secondary transmitters. Then, computer simulations employing the Monte-Carlo approach are realized to validate the derivations.
\end{abstract}

\section{Introduction}

Multihop relaying protocols [1-5] are commonly used to enhance performance for wireless networks, e.g., wireless sensor networks (WSNs) and wireless ad hoc networks (WANs). In WSNs and WANs, the transmit power of wireless devices is often limited, and hence, the source cannot directly communicate with the destination at a far distance. In multihop decode-and-forward protocol (M-DF-P) [6-8], intermediate nodes (or relays) decode the received signals, reencode, and send the encoded ones to next hop. In multihop amplify-and-forward protocol (M-AF-P) [9-11], relays amplify the received signals and forward the amplified ones to a next node. The implementation of M-DF-P is more complex than that of M-AF-P, but M-DF-P obtains better end-to- end (e2e) performance due to the decoding process at each hop. As shown in $[12,13]$, M-DF-P outperforms M-AF-P over fading and limited cochannel interference (CCI) environments, in terms of outage probability (OP) and bit error rate (BER). References $[14,15]$ evaluated the e2e OP of MAF-P in a random presence of interference sources. In [16], the authors studied the impact of I/Q imbalance on the performance of M-AF-P. In [17], joint impact of hardware impairments (HI) and CCI on the e2e OP and average channel capacity of M-DF-P was studied. As shown in [17], in fading and CCI environments, the performance of multihop relaying protocols is significantly degraded.

To enhance the performance for multihop relaying protocols over fading environments, various diversity methods have been proposed in $[18-26]$. The authors of $[18,19]$ 
introduced cooperative multihop protocols, where the relays can exploit the broadcast nature of wireless medium to obtain spatial diversity. Particularly, the relays attempt to receive the signals from all the previous nodes and process them appropriately to enhance reliability of the data decoding. Different with $[18,19]$, diversity-based multihop transmission protocols in $[20,21]$ perform cooperative communication at each hop by using external nodes that are not on the source-destination route. In [22, 23], relay selection approaches in cluster-based multihop transmission networks were studied. In the system models proposed in $[22,23]$, the source data is transmitted to the destination via intermediate clusters. Moreover, opportunistic relay selection methods are performed at each cluster to attain the diversity gain. In [24-26], the authors introduced ultradense wireless networks in which there exist multiple available paths between a source and a destination. In addition, path selection strategies were proposed to obtain better performance.

Cognitive radio (CR) [27] was first proposed by J. Mitola in 1999 to solve the spectrum scarcity issue and inefficient spectrum usage. In CR, primary users who are used licensed bands at any time can share spectrum to secondary users. Underlay cognitive radio (UCR) [28-30] is one of efficient spectrum-sharing methods that can guarantee the continuous data transmission for the secondary network. However, the secondary transmitters in the UCR networks must adjust their transmit power so that quality of service $(\mathrm{QoS})$ of the primary network is not harmful. In [31], the authors considered the performance of multihop UCR AF relaying protocols over the Nakagami-m fading channels. In $[32,33]$, the authors proposed M-DF-P in the UCR networks, where the secondary transmitters can harvest wireless energy from a power beacon deployed in the secondary network. References $[34,35]$ proposed cluster-based multihop UCR protocols using the DF technique and relay selection methods at each cluster. A best-path selection method was studied in [36] to improve the e2e performance of the UCR networks under the interference constraint and presence of an active eavesdropper.

Recently, nonorthogonal multiple access (NOMA) [3739] has gained much attention as a promising method to improve data rate for wireless systems. In downlink NOMA scenarios $[40,41]$, a base station linearly combines signals with different transmit power levels before transmitting them to intended users at the same time, frequency, and code. At these users, the successive interference cancellation (SIC) technique can be performed to obtain their desired signals. For uplink NOMA methods [42, 43], the users can simultaneously send their signals to the base station. Similarly, different transmit power levels have to be used at the users so that the base station can realize SIC for decoding the received signals. References [44-46] studied the outage performance of dual-hop relaying protocols in the UCR networks. The authors in [47] proposed multihop energy-harvesting WSNs employing NOMA and cooperative jamming methods to enhance the e2e secrecy performance.

In this paper, we propose best-path selection methods to enhance the OP performance for both M-DF-P and M-AF-P in the UCR networks under the joint impact of CCI from primary transmitters and HI. In particular, a secondary source selects one of available paths providing maximum instantaneous e2e channel capacity to send its data to a secondary destination. We also propose an uplink NOMA method to increase the data rate for the primary network, i.e., multiple primary transmitters send their data to a primary receiver at the same time. Different with $[45,46]$, we consider multihop DF and AF UCR networks where the primary network uses NOMA to enhance the system throughput. Unlike [34,35], this paper concerns with path selection strategies in the UCR networks (see Table 1). Although various path selection methods were proposed in [24-26], these published works did not consider the UCR networks. Moreover, unlike [24-26, 34, 35], the joint impact of CCI and $\mathrm{HI}$ is studied in this paper. Although references $[17,48]$ evaluated the performance of M-DF-P in the presence of the limited interference and hardware noises, the authors in [17] only considered a single-path scheme, and reference [48] did not consider the UCR environment. In addition, our proposed models consider both $\mathrm{DF}$ and AF protocols, while only M-DF-P is studied in $[17,48]$. Unlike [36], we propose the uplink NOMA scheme for the primary network. Furthermore, reference [36] assumed that the transceiver hardware of all wireless devices is perfect, and CCI from the primary transmitter to secondary receivers can be relaxed.

The main contribution of this paper can be listed as follows:

(i) Firstly, the NOMA approach is proposed to provide a higher data rate for the primary network. Moreover, we also propose an efficient method to allocate transmit power for the secondary transmitters to guarantee QoS for the primary network, i.e., the secondary transmitters have to adjust their transmit power so that the outage performance of the primary network is lower than a predetermined threshold

(ii) Secondly, the best-path selection methods are proposed to enhance the e2e OP for the secondary networks under the joint impact of the interference constraint, CCI from the primary transmitters, and Rayleigh fading channel. In addition, we consider a practical scenario, i.e., the secondary nodes are low-cost devices (e.g., sensor nodes), and hence, their transceiver hardware may be not perfect

(iii) Next, we derive an exact closed-form expression of OP for the primary network. The e2e OP of M-DF$\mathrm{P}$ and M-AF-P of the secondary networks is also analyzed. Particularly, we derive the exact and asymptotic closed-form formulas of the e2e OP for $\mathrm{M}-\mathrm{DF}-\mathrm{P}$, and lower-bound ones of the e2e OP for M-AF-P

(iv) Finally, the Monte-Carlo based computer simulations are realized to validate the derived formulas as well as to compare the e2e OP performance of M-DF-P and M-AF-P. 
TABLE 1: Main differences with the published works related to multihop relaying networks.

\begin{tabular}{lccccc}
\hline References & Underlay CR & NOMA-based primary transmission & Path selection & Joint impact of CCI and HI & DF/AF protocol \\
\hline Proposed scheme & Yes & Yes & Yes & Yes & Both \\
Reference [17] & No & No & No & Yes & Only DF \\
Reference [24,25] & No & No & Yes & No & Only DF \\
Reference [26] & No & No & Yes & Only HI & Only DF \\
Reference [34,35] & Yes & No & No & No & Only DF \\
Reference [36] & Yes & No & Yes & No & Only DF \\
Reference [48] & No & No & Yes & Yes & Only DF \\
\hline
\end{tabular}

The rest of this paper is organized as follows. The system model of multihop multipath UCR networks is described in "System Model." Performance evaluation is given in "Performance Analysis." "Numerical Results" presents both simulation and analytical results to compare performance of M-DF-P and M-AF-P. Finally, conclusions are given in "Conclusion."

\section{System Model}

Figure 1 illustrates the system model of the proposed M-DF$\mathrm{P}$ and M-AF-P in the UCR networks. In the primary network, $N$ primary transmitters $\left(\mathrm{PT}_{n}, n=1,2, \cdots, N\right)$ attempt to send their data to a primary receiver (PR). To enhance the throughput for the primary network, the primary transmitters at the same time send their data to the PR node by using NOMA. In addition, the PT nodes use different transmit power levels, and the PR node uses SIC to decode the received data. In the secondary network, a source $(S)$ wants to transmit its data to a destination (D). Because the direct link between $S$ and $\mathrm{D}$ does not exist, the multihop relaying technique is employed to relay the source data to the destination. Assume that there are $M$ available paths between $S$ and $\mathrm{D}(M \geq 1)$, and one of them is selected for the data transmission. Let $L_{m}$ denote the number of relay nodes on the $m$ th path, where $m=1,2, \cdots, M, L_{m} \geq 1$. As depicted in Figure 1, the relays on the $m$ th path are named by $R_{m, 1}, R_{m, 2}, \ldots$, $R_{m, L m}$. For ease of presentation and analysis, we also denote $S$ and D by $R_{m, 0}$ and $R_{m, L m+1}(\forall m)$, respectively. Assume that all the nodes $X$ have only a single antenna and operate on a half-duplex mode, where $X \in\left\{R_{m, v}, P T_{n}, P R\right\}, v=0,1, \cdots$, $L_{m}+1$.

Let us denote $h_{X, Y}$ as the Rayleigh fading channel coefficient between $X$ and $Y$, where $(X, Y) \in\left\{R_{m, v}, P T n, P R\right\}$. Hence, channel gain, i.e., $\varphi_{X, Y}=\left|h_{X, Y}\right|^{2}$, is an exponential random variable $(\mathrm{RV})$ whose distributions are given as

$$
F_{\varphi X, Y}(x)=1-\exp \left(-\lambda_{X, Y} x\right), f_{\varphi X, Y}(x)=\lambda_{X, Y} \exp \left(-\lambda_{X, Y} x\right)
$$

where $F_{U}($.$) and f_{U}($.$) are cumulative distribution function$ (CDF) and probability density function (PDF) of a RV $U$, respectively, $\lambda_{\mathrm{X}, \mathrm{Y}}=1 / \varepsilon\left\{\varphi_{\mathrm{X}, \mathrm{Y}}\right\}=d_{\mathrm{X}, \mathrm{Y}}^{P L} \quad[34-36], \varepsilon\{\bullet\}$ is an

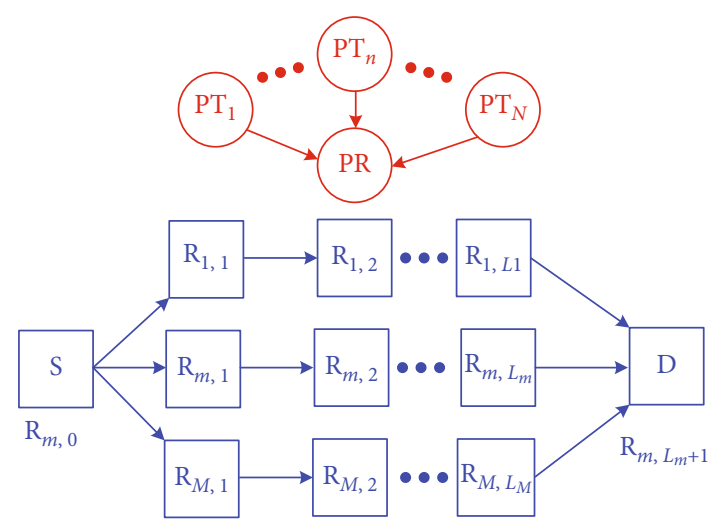

FIGURE 1: System model of multihop DF/AF relaying protocols in the UCR networks.

expected operator, $d_{X, Y}$ is distance between $X$ and $Y$, and $P L(2 \leq P L \leq 6)$ is a path-loss exponent.

2.1. Hardware Impairments. Considering the data transmission between a transmitter $X$ and a receiver $Y$, under the impact of HI (assume that there is no CCI), the signal received by $Y$ is written as

$$
y_{\mathrm{Y}}=\sqrt{P_{\mathrm{X}}} h_{\mathrm{X}, \mathrm{Y}}\left(s_{\mathrm{X}}+\eta_{\mathrm{X}, \mathrm{Y}}\right)+n_{\mathrm{Y}}
$$

where $P_{\mathrm{X}}$ is transmit power of $X, s_{X}$ is signal of $X, \eta_{X, Y}$ is the hardware noise caused by the hardware imperfection at $X$ and $Y$, and $n_{Y}$ is the additive white Gaussian noise (AWGN) at $Y$ with zero-mean. Similar to $[49,50], \eta_{X, Y}$ can be modeled as a zero-mean Gaussian RV whose variance is $\kappa_{\mathrm{X}, \mathrm{Y}}^{2},\left(\kappa_{\mathrm{X}, \mathrm{Y}}^{2}\right.$ is called to be total hardware impairment level of the $X \longrightarrow Y$ link).

2.1.1. Assumption and Notation. Assume that variance of AWGN at all of the receivers is same, i.e., $\sigma_{0}^{2}$. It is assumed that the transceiver hardware of the primary terminals is perfect, i.e., $\kappa_{\mathrm{PT}_{n}, \mathrm{PR}}^{2}=0(\forall n)$. Next, we denote $\kappa_{s s}^{2}, \kappa_{s p}^{2}$, and $\kappa_{p s}^{2}$ as the total hardware impairment levels of the $\mathrm{R}_{m, v} \longrightarrow$ $\mathrm{R}_{m, v+1}, \mathrm{R}_{m, v} \longrightarrow \mathrm{PR}$, and $\mathrm{PT}_{n} \longrightarrow \mathrm{R}_{m, v}$ links, respectively, for all $m, n, v$.

2.2. Operation Principle. In this subsection, assume that the $m$ th path is selected, and hence, the data transmission is split into $\left(L_{m}+1\right)$ orthogonal time slots. At the first time slot, the 
source $\left(R_{m, 0}\right)$ sends its data to $R_{m, 1}$, while the primary transmitters simultaneously transmit their data to PR.

(1) Primary network: under the joint impact of $\mathrm{CCI}$ and $\mathrm{HI}$, the received signal at PR in this time slot can be given as

$y_{\mathrm{PR}}^{(1)} \sum_{n=1}^{N} \sqrt{\beta_{n} P_{\mathrm{PT}}} h_{\mathrm{PT}_{n}, \mathrm{PR}} s_{\mathrm{PT}_{n}}^{(1)}+\sqrt{P_{\mathrm{R}_{m, 0}}} h_{\mathrm{R}_{m, 0}, \mathrm{PR}}\left(s_{S}+\eta_{\mathrm{R}_{m, 0}, \mathrm{PR}}\right)+n_{\mathrm{PR}}^{(1)}$.

In (3), $s_{S}$ is the signal of the source, $s_{\mathrm{PT}_{n}}^{(1)}$ is the signal of $P T_{n}$ in the first time slot, $P_{\mathrm{R}_{m, 0}}$ is the transmit power of the source, and $P_{\mathrm{PT}}$ is the maximum transmit power of all the primary transmitters. Following the NOMA principle, the primary transmitters must use different transmit powers so that $\mathrm{PR}$ can decode the received signals. As presented in (3), $\beta_{n} P_{\mathrm{PT}}$ is the transmit power of $P T_{n}$, where $\beta_{1}=1, \beta_{1}>$ $\beta_{2}>\ldots>\beta_{N}>0$. With this power allocation method, PR will decode the signal $s_{\mathrm{PT}_{n}}^{(1)}$, next uses SIC to remove the component $\sqrt{\beta_{n} P_{\mathrm{PT}}} h_{\mathrm{PT}_{n}, \mathrm{PR}_{\mathrm{PT}}} s_{\mathrm{PT}_{n}}^{(1)}$ from $y_{\mathrm{PR}}^{(1)}$, and then decodes $s_{\mathrm{PT}_{n+1}}^{(1)}$. Indeed, if the SIC operation is perfect, the signal used for decoding $s_{\mathrm{PT}_{n+1}}^{(1)}$ can be rewritten as

$z_{\mathrm{PR}, n+1}^{(1)}=\sum_{k=n+1}^{N} \sqrt{\beta_{k} P_{\mathrm{PT}}} h_{\mathrm{PT}_{k}, \mathrm{PR}_{\mathrm{PT}}} s_{k}^{(1)}+\sqrt{P_{\mathrm{R}_{m, 0}}} h_{\mathrm{R}_{m, 0}, \mathrm{PR}}\left(s_{S}+\eta_{\mathrm{R}_{m, 0}, \mathrm{PR}}\right)+n_{\mathrm{PR}}^{(1)}$,

where we note that $z_{\mathrm{PR}, 1}^{(1)} \equiv y_{\mathrm{PR}}^{(1)}$.

For the last signal $s_{\mathrm{PT}_{N}}^{(1)}$, Equation (4) reduces to

$z_{\mathrm{PR}, N}^{(1)}=\sqrt{\beta_{N} P_{\mathrm{PT}}} h_{\mathrm{PT}_{N}, \mathrm{PR}_{\mathrm{PT}}} s_{\mathrm{PT}_{N}}^{(1)}+\sqrt{P_{\mathrm{R}_{m, 0}}} h_{\mathrm{R}_{m, 0}, \mathrm{PR}}\left(s_{S}+\eta_{\mathrm{R}_{m, 0}, \mathrm{PR}}\right)+n_{\mathrm{PR}}^{(1)}$.

Combining (3), (4), and (5), the signal-to-interferenceplus-noise (SINR) obtained by PR for decoding $s_{\mathrm{PT}_{n}}^{(1)}$ is obtained as

$\gamma_{\mathrm{PR}, \mathrm{s}_{\mathrm{PT}}}^{(1)}=\left\{\begin{array}{l}\frac{\beta_{n} P_{\mathrm{PT}} \varphi_{\mathrm{PT}_{n}, \mathrm{PR}}}{\sum_{u=n+1}^{N} \beta_{u} P_{\mathrm{PT}_{\mathrm{T}}} \varphi_{\mathrm{PT}_{u}, \mathrm{PR}}+\left(1+\kappa_{\mathrm{sp}}^{2}\right) P_{\mathrm{R}_{m, 0}} \varphi_{\mathrm{R}_{m, 0}, \mathrm{PR}}+\sigma_{0}^{2}}, n<N \\ \frac{\beta_{N} P_{\mathrm{PT}_{\mathrm{PT}}} \varphi_{\mathrm{PT}_{N}, \mathrm{PR}}}{\left(1+\kappa_{\mathrm{sp}}^{2}\right) P_{\mathrm{R}_{m, 0}} \varphi_{\mathrm{R}_{m, 0}, \mathrm{PR}}+\sigma_{0}^{2}}, n=N .\end{array}\right.$

(2) Secondary network: considering the secondary network at the first time slot, the received signal at $R_{m, 1}$ can be expressed as

$$
\begin{aligned}
y_{\mathrm{R}_{m, 1}}= & \sqrt{P_{\mathrm{R}_{m, 0}}} h_{\mathrm{R}_{m, 0}, \mathrm{R}_{m, 1}}\left(s_{S}+\eta_{\mathrm{R}_{m, 0}, \mathrm{R}_{m, 1}}\right)+\sum_{n=1}^{N} \sqrt{\beta_{n} P_{\mathrm{PT}}} h_{\mathrm{PT}_{n}, \mathrm{R}_{m, 1}} \\
& \cdot\left(s_{\mathrm{PT}_{n}}^{(1)}+\eta_{\mathrm{PT}_{n}, \mathrm{R}_{m, 1}}\right)+n_{\mathrm{R}_{m, 1}} .
\end{aligned}
$$

From (7), the SINR received by $R_{m, 1}$ is calculated as

$$
\gamma_{\mathrm{R}_{m, 0}, \mathrm{R}_{m, 1}}=\frac{P_{\mathrm{R}_{m, 0}} \varphi_{\mathrm{R}_{m, 0}, \mathrm{R}_{m, 1}}}{\kappa_{s s}^{2} P_{\mathrm{R}_{m, 0}} \varphi_{\mathrm{R}_{m, 0}, \mathrm{R}_{m, 1}}+\left(1+\kappa_{\mathrm{ps}}^{2}\right) \sum_{n=1}^{N} \beta_{n} P_{\mathrm{PT}_{\mathrm{T}}} \varphi_{\mathrm{PT}_{n}, \mathrm{R}_{m, 1}}+\sigma_{0}^{2}} .
$$

Next, we describe the operation of the proposed M-DF-P and M-AF-P models at next time slots.

(3) M-DF-P: in this protocol, $R_{m, 1}$ decodes $s_{S}$, encodes again, and then forwards it to $R_{m, 2}$ in the second time slot. Generally, at the $t$ th time slot, $R_{m, t-1}$ sends $s_{S}$ to $R_{m, t}$, and the received signal at PR and $R_{m, t}$ can be formulated, respectively, as

$$
\begin{aligned}
y_{\mathrm{PR}, \mathrm{DF}}^{(t)}= & \sum_{n=1}^{N} \sqrt{\beta_{n} P_{\mathrm{PT}}} h_{\mathrm{PT}_{n}, \mathrm{PR}} s_{\mathrm{PT}_{n}}^{(t)}+\sqrt{P_{\mathrm{R}_{m, t-1}}} h_{\mathrm{R}_{m, t-1}, \mathrm{PR}} \\
& \cdot\left(s_{S}+\eta_{\mathrm{R}_{m, t-1}, \mathrm{PR}}\right)+n_{\mathrm{PR}}^{(t)}, \\
y_{\mathrm{R}_{m, t} \mathrm{DF}}= & \sqrt{P_{\mathrm{R}_{m, t-1}}} h_{\mathrm{R}_{m, t-1}, \mathrm{R}_{m, t}}\left(s_{S}+\eta_{\mathrm{R}_{m, t-1}, \mathrm{R}_{m, t}}\right) \\
& +\sum_{n=1}^{N} \sqrt{\beta_{n} P_{\mathrm{PT}}} h_{\mathrm{PT}_{n}, \mathrm{R}_{m, t}}\left(s_{\mathrm{PT}_{n}}^{(t)}+\eta_{\mathrm{PT}_{n}, \mathrm{R}_{m, t}}\right)+n_{R_{m, t}},
\end{aligned}
$$

where $t=1,2, \cdots, L_{m}+1, s_{\mathrm{PT}_{n}}^{(t)}$ is the signal of $\mathrm{PT}_{n}$ in this time slot and $P_{R_{m, t-1}}$ is the transmit power of $R_{m, t-1}$.

Similar to the first time slot, PR uses SIC to decode all the data. Then, the SINR obtained at PR, with respect to $s_{\mathrm{PT}_{n}}^{(t)}$, is shown as

$\gamma_{s_{\mathrm{PT}_{n}}}^{(t)}=\left\{\begin{array}{l}\frac{\beta_{n} P_{\mathrm{PT}} \varphi_{\mathrm{PT}_{n}, \mathrm{PR}}}{\sum_{u=n+1}^{N} \beta_{u} P_{\mathrm{PT}_{\mathrm{T}}} \varphi_{\mathrm{PT}_{u}, \mathrm{PR}}+\left(1+\kappa_{\mathrm{sp}}^{2}\right) P_{\mathrm{R}_{m, t-1}} \varphi_{\mathrm{R}_{m, t-1}, \mathrm{PR}}+\sigma_{0}^{2}}, n<N \\ \frac{\beta_{N} P_{\mathrm{PT}} \varphi_{\mathrm{PT}_{N}, \mathrm{PR}}}{\left(1+\kappa_{\mathrm{sp}}^{2}\right) P_{\mathrm{R} t-1} \varphi_{\mathrm{R}_{m, t-1}, \mathrm{PR}}+\sigma_{0}^{2}}, n=N .\end{array}\right.$

Using (10), the SINR at $R_{m, t}$ can be given as follows:

$$
\gamma_{\mathrm{R}_{m, t-1}, \mathrm{R}_{m, t}}=\frac{P_{\mathrm{R}_{m, t-1}} \varphi_{\mathrm{R}_{m, t-1}, \mathrm{R}_{m, t}}}{\kappa_{s s}^{2} P_{\mathrm{R}_{m, t-1}} \varphi_{\mathrm{R}_{m, t-1}, \mathrm{R}_{m, t}}+\left(1+\kappa_{\mathrm{ps}}^{2}\right) \sum_{n=1}^{N} \beta_{n} P_{\mathrm{PT}_{\mathrm{T}}} \varphi_{\mathrm{PT}_{n}, \mathrm{R}_{m, t}}+\sigma_{0}^{2}} .
$$

Due to usage of the DF relaying method, the e2e channel capacity of the $m$ th path can be expressed as 


$$
C_{m}^{e 2 e, \mathrm{DF}}=\frac{1}{L_{m}+1} \log _{2}\left(1+\min _{t=1,2, \cdots, L_{m+1}}\left(\gamma_{\mathrm{R}_{m, t-1}, \mathrm{R}_{m, t}}\right)\right),
$$

where the factor $1 /(L m+1)$ implies that the data transmission of the secondary network is split into $\left(L_{m}+1\right)$ time slots.

(4) M-AF-P: in M-AF-P, $R_{m, 1}$ amplifies the received signal $y_{R_{m, 1}}$, i.e., $z_{R_{m, 1}}=G_{R_{m, 1}} y_{R_{m, 1}}$, and sends $z_{R_{m, 1}}$ to $R_{m, 2}$ in the second time slot. Because $P_{\mathrm{R}_{m, 1}}=G_{\mathrm{R}_{m, 1}}^{2} \varepsilon$ $\left\{\left|y_{\mathrm{R}_{m, 1}}\right|^{2}\right\}$, using (7), the amplify factor $G_{\mathrm{R} m, 1}$ can be determined as

$$
G_{\mathrm{R}_{m, 1}}=\sqrt{\frac{P_{\mathrm{R}_{m, 1}}}{\left(1+\kappa_{s s}^{2}\right) P_{\mathrm{R}_{m, 0}} \varphi_{\mathrm{R}_{m, 0}, \mathrm{R}_{m, 1}}+\left(1+\kappa_{\mathrm{ps}}^{2}\right) \sum_{n=1}^{N} \beta_{n} P_{\mathrm{PT}} \varphi_{\mathrm{PT}_{m}, \mathrm{R}_{m, 1}}+\sigma_{0}^{2}}} .
$$

Next, the received signal at $\mathrm{PR}$ and $R_{m, 2}$ can be expressed, respectively, as

$$
y_{\mathrm{PR}, \mathrm{AF}}^{(2)}=\sum_{n=1}^{N} \sqrt{\beta_{n} P_{\mathrm{PT}}} h_{\mathrm{PT}_{n}, \mathrm{PR}} s_{\mathrm{PT}_{n}}^{(2)}+h_{\mathrm{R}_{m, 1}, \mathrm{PR}}\left(z_{\mathrm{R}_{m, 1}}+\eta_{\mathrm{R}_{m, 1}, \mathrm{PR}}\right)+n_{\mathrm{PR}}^{(2)},
$$

$$
\begin{aligned}
y_{\mathrm{R}_{m, 2}, \mathrm{AF}}= & h_{\mathrm{R}_{m, 1}, \mathrm{R}_{m, 2}}\left(z_{\mathrm{R}_{m, 1}}+\eta_{\mathrm{R}_{m, 1}, \mathrm{R}_{m, 2}}\right)+\sum_{n=1}^{N} \sqrt{\beta_{n} P_{\mathrm{PT}_{1}}} h_{\mathrm{PT}_{n}, \mathrm{R}_{m, 2}} \\
& \cdot\left(s_{\mathrm{PT}_{n}}^{(2)}+\eta_{\mathrm{PT}_{n}, \mathrm{R}_{m, 2}}\right)+n_{\mathrm{R}_{m, 2}} .
\end{aligned}
$$

Similar to [49], variances of $\eta_{R m, 1, P R}$ and $\eta_{R m, 1, R m, 2}$ can be calculated, respectively, as

$$
\begin{gathered}
\operatorname{Var}\left\{\eta_{\mathrm{R}_{m, 1}, \mathrm{PR}}\right\}=\kappa_{\mathrm{sp}}^{2} G_{\mathrm{R}_{m, 1}}^{2} \varepsilon\left\{\left|y_{\mathrm{R}_{m, 1}}\right|^{2}\right\}=\kappa_{\mathrm{sp}}^{2} P_{\mathrm{R}_{m, 1}}, \\
\operatorname{Var}\left\{\eta_{\mathrm{R}_{m, 1}, \mathrm{R}_{m, 2}}\right\}=\kappa_{s s}^{2} G_{\mathrm{R}_{m, 1}}^{2} \varepsilon\left\{\left|y_{\mathrm{R}_{m, 1}}\right|^{2}\right\}=\kappa_{s s}^{2} P_{\mathrm{R}_{m, 1}} .
\end{gathered}
$$

Combining (15) and (17), the SINR obtained at PR, with respect to $s_{\mathrm{PT}_{n}}^{(2)}$, is given as

$$
\gamma_{\mathrm{sP}_{n}}^{(2)}=\left\{\begin{array}{l}
\frac{\beta_{n} P_{\mathrm{PT}} \varphi_{\mathrm{PT}_{n}, \mathrm{PR}}}{\sum_{u=n+1}^{N} \beta_{u} P_{\mathrm{PT}_{\mathrm{T}}} \varphi_{\mathrm{PT}_{u}, \mathrm{PR}}+\left(1+\kappa_{\mathrm{sp}}^{2}\right) P_{\mathrm{R}_{m, 1}} \varphi_{\mathrm{R}_{m, 1}, \mathrm{PR}}+\sigma_{0}^{2}}, n<N \\
\frac{\beta_{N} P_{\mathrm{PT}_{\mathrm{T}}} \varphi_{\mathrm{PT}_{N}, \mathrm{PR}}}{\left(1+\kappa_{\mathrm{sp}}^{2}\right) P_{\mathrm{R}_{m, 1}} \varphi_{\mathrm{R}_{m, 1} \mathrm{PR}}+\sigma_{0}^{2}}, n=N .
\end{array}\right.
$$

Next, combining (7), (16), and (18), we can formulate the SINR received at $R_{m, 2}$ by

$$
\Psi_{\mathrm{R}_{m, 2}}=\frac{\gamma_{\mathrm{R}_{m, 0}, \mathrm{R}_{m, 1}} \gamma_{\mathrm{R}_{m, 1}, \mathrm{R}_{m, 2}}}{\gamma_{\mathrm{R}_{m, 0}, \mathrm{R}_{m, 1}}+\gamma_{\mathrm{R}_{m, 1}, \mathrm{R}_{m, 2}}+1},
$$

where it is worth noting that $\gamma_{\mathrm{R} m, 0, \mathrm{R} m, 1}$ and $\gamma_{\mathrm{R} m, 1, \mathrm{R} m, 2}$ are given as in (12).

Similarly, $\mathrm{R}_{m, 2}$ amplifies $y_{\mathrm{R} m, 2, \mathrm{AF}}$ and then forwards the amplified signal to $\mathrm{R}_{m, 3}$ in the third time slot. Using an inductive method, we can obtain an exact formula of the SINR obtained at $\mathrm{R}_{m, t}$ at the $t$ th time slot as follows:

$$
\Psi_{\mathrm{R}_{m, t}}=\frac{\prod_{k=1}^{t} \gamma_{\mathrm{R}_{m, k-1}, \mathrm{R}_{m, k}}}{\prod_{k=1}^{t}\left(1+\gamma_{\mathrm{R}_{m, k-1}, \mathrm{R}_{m, k}}\right)-\prod_{k=1}^{t} \gamma_{\mathrm{R}_{m, k-1}, \mathrm{R}_{m, k}}},
$$

where $1<t \leq L_{m+1}$. Then, the e2e channel capacity of the $m$ th path can be computed as

$$
\begin{aligned}
C_{m}^{e 2 e, \mathrm{AF}=} & \frac{1}{L_{m}+1} \log _{2}\left(1+\Psi_{\mathrm{R}_{m, L_{m}+1}}\right)=\frac{1}{L_{m}+1} \log _{2} \\
& \cdot\left(1+\frac{\prod_{k=1}^{L_{m}+1} \gamma_{\mathrm{R}_{m, k-1}, \mathrm{R}_{m, k}}}{\prod_{k=1}^{L_{m}+1}\left(1+\gamma_{\mathrm{R}_{m, k-1}, \mathrm{R}_{m, k}}\right)-\prod_{k=1}^{L_{m}+1} \gamma_{\mathrm{R}_{m, k-1}, \mathrm{R}_{m, k}}}\right) .
\end{aligned}
$$

Remark 1. In both M-DF-P and M-AF-P, the SINRs obtained at $P R$ at the $t$ th time slot is the same (see (11)). Therefore, the channel capacity of the $P T n \longrightarrow P R$ link at the $t$ th hop of the $m$ th path is shown as

$$
C_{s_{\mathrm{PT}_{n}}}^{(t)}=\frac{1}{L_{m}+1} \log _{2}\left(1+\gamma_{s_{\mathrm{PT}_{n}}}^{(t)}\right)
$$

2.3. Best-Path Selection Methods. To maximize the e2e channel capacity, we propose the best-path selection methods for M-DF-P and M-AF-P as follows:

$$
C_{b}^{e 2 e, Z}=\max _{m=1,2, \cdots, M}\left(C_{m}^{e 2 e, Z}\right),
$$

where $b(b \in\{1,2, \cdots, M\})$ denotes the best path, and $Z \in\{$ $\mathrm{DF}, \mathrm{AF}\}$.

Remark 2. To select the best path, the secondary nodes on the paths have to estimate the channel state information (CSI) of the data links and the interference links. In addition, the secondary transmitters including source and relays also have to know their transmit power (see "Transmit Power of Secondary Transmitters"). Via operation of the routing set-up and maintenance, the secondary nodes can exchange the estimated CSIs and their transmit power together. Therefore, the source can obtain information of the instantaneous SINR (and instantaneous channel capacity as well) of all the hops so that it can select the best path by using the algorithm given in (24). It is also assumed that the operation of the CSI estimation is perfect, and the channels between the nodes are slow Rayleigh fading, which remain unchanged in each the source-destination data transmission. 


\section{Performance Analysis}

This section evaluates OP of the primary and secondary networks. We also derive expressions of the transmit power for the secondary transmitters to guarantee QoS for the primary network.

3.1. OP of Primary Network. When the $m$ th path is selected, let us consider the minimum channel capacity between $\mathrm{PT}_{n}$ and PR at the $t$ th time slot:

$$
C_{\mathrm{PR}, \min }^{(t)}=\min _{n=1,2, \cdots, N}\left(C_{{\mathrm{P}_{\mathrm{P}}}_{n}}^{(t)}\right)=\frac{1}{L_{m}+1} \log _{2}\left(1+\min _{n=1,2, \cdots, N}\left(\gamma_{s_{\mathrm{PT}_{n}}}^{(t)}\right)\right) .
$$

We now define OP of the primary network as the probability that $C_{\mathrm{PR}, \min }^{(t)}$ is lower than a positive threshold, i.e., $C_{\mathrm{P}, \mathrm{th}}$, and is expressed as

$$
\mathrm{OP}_{P R}^{(t)}=\operatorname{Pr}\left(C_{\mathrm{PR}, \min }^{(t)}<C_{\mathrm{P}, t h}\right)=\operatorname{Pr}\left(\min _{n=1,2, \cdots, N}\left(\gamma_{\mathrm{PT}_{n}}^{(t)}\right)<\theta_{\mathrm{P}, m}\right) \text {, }
$$

where

$$
\theta_{P, m}=2^{(L m+1) C P, t h}-1 .
$$

Equation (26) also implies that the primary network is in outage if one of the signals $s_{\mathrm{PT}_{n}}^{(t)}$ cannot be correctly decoded. In particular, employing the independence of RVs $\gamma_{\mathrm{PT}_{n}}^{(t)}$, Equation (26) can be rewritten as

$$
\mathrm{OP}_{\mathrm{PR}}^{(t)}=1-\prod_{n=1}^{N}\left(1-\operatorname{Pr}\left(\gamma_{\mathrm{PT}_{n}}^{(t)}<\theta_{\mathrm{P}, m}\right)\right)
$$

where $\operatorname{Pr}\left(\gamma_{\mathrm{PT}_{n}}^{(t)}<\theta_{\mathrm{P}, m}\right)$ is the probability that the decoding of $s_{\mathrm{PT}_{n}}^{(t)}$ fails.

Combining (11) and (28) yields (29) as

$$
\begin{aligned}
\operatorname{Pr}\left(\gamma_{s_{\mathrm{PT}_{n}}}^{(t)}<\theta_{\mathrm{P}, m}\right)= & \int_{0}^{+\infty} \ldots \int_{0}^{+\infty} F_{\varphi_{\mathrm{PT}_{n}, \mathrm{PR}}}\left(\sum_{u=n+1}^{N} a_{u, n} x_{u}+b_{n} \tau_{m, t-1} y+c_{n}\right) \\
& \times f_{\varphi_{\mathrm{R}_{m}, t-1, \mathrm{PR}}}(y) d y \prod_{u=n+1}^{N} f_{\varphi_{\mathrm{PT}_{u}, \mathrm{PR}}}\left(x_{u}\right) d x_{u},
\end{aligned}
$$

where

$$
a_{u, n}=\left\{\begin{array}{l}
\beta_{u} \theta_{\mathrm{P}, m} / \beta_{n}, n<N \\
0, n=N
\end{array}, b_{n}=\frac{\left(1+\kappa_{\mathrm{sp}}^{2}\right) \theta_{\mathrm{P}, m}}{\beta_{n}}, c_{n}=\frac{\sigma_{0}^{2} \theta_{\mathrm{P}, m}}{\beta_{n} P_{\mathrm{PT}}}, \tau_{m, t-1}=\frac{P_{\mathrm{R}_{m, t-1}}}{P_{\mathrm{PT}}} .\right.
$$

Substituting CDF and PDF given by (1) into (29), after some manipulation, we obtain

$$
\operatorname{Pr}\left(\gamma_{s_{\mathrm{PT}_{n}}}^{(t)}<\theta_{\mathrm{P}, m}\right)=1-\frac{g_{u, n}}{l_{n}+\tau_{m, t-1}}
$$

where

$$
\begin{aligned}
g_{u, n} & =\left[\prod_{u=n+1}^{N} \frac{\lambda_{\mathrm{PT}_{u}, \mathrm{PR}}}{\lambda_{\mathrm{PT}_{u}, \mathrm{PR}}+a_{u, n} \lambda_{\mathrm{PT}_{n}, \mathrm{PR}}}\right] \frac{\lambda_{\mathrm{R}_{m, t-1}, \mathrm{PR}}}{b_{n} \lambda_{\mathrm{PT}_{n}, \mathrm{PR}}} \exp \left(-\lambda_{\left.\mathrm{PT}_{n}, \mathrm{PR}_{n} c_{n}\right), l_{n}}\right. \\
& =\frac{\lambda_{\mathrm{R}_{m, t-1}, \mathrm{PR}}}{b_{n} \lambda_{\mathrm{PT}_{n}, \mathrm{PR}}} .
\end{aligned}
$$

Substituting (31) into (28), OP of the primary network at the $t$ th time slot can be expressed by an exact closed-form expression as follows:

$$
\mathrm{OP}_{\mathrm{PR}}^{(t)}=1-\prod_{n=1}^{N} \frac{g_{u, n}}{l_{n}+\tau_{m, t-1}}
$$

3.2. Transmit Power of Secondary Transmitters. At first, QoS of the primary network in the $t$ th time slot is given as

$$
\mathrm{OP}_{\mathrm{PR}}^{(t)} \leq \varepsilon_{\mathrm{OP}}
$$

where $\varepsilon_{\mathrm{OP}}$ is an allowable maximum value of OP at any time slot, and it is an important design parameter of the primary network.

Hence, the transmit power of $\mathrm{R}_{m, t-1}$ can be obtained by solving equation $\mathrm{OP}_{\mathrm{PR}}^{(t)}=\varepsilon_{\mathrm{OP}}$. More particularly, we have to find a positive real solution (denoted by $\tau_{m, t-1}^{*}$ ) of the following equation:

$$
\prod_{n=1}^{N}\left(\tau_{m, t-1}+l_{n}\right)=\frac{1}{1-\varepsilon_{\mathrm{OP}}} \prod_{n=1}^{N} g_{u, n}
$$

Then, the transmit power of $\mathrm{R}_{m, t-1}$ is obtained as $P_{\mathrm{R}_{m, t-1}}$ $=P_{\mathrm{PT}} \tau_{m, t-1}^{*}$. In case that Equation (35) has no positive real solution, we have $P_{\mathrm{R} m, t-1}=0$, which means $\mathrm{R}_{m, t-1}$ is not allowed to access the licensed band.

For example, when $N=2, \tau_{m, t-1}^{*}$ is a solution of (36):

$$
\tau_{m, t-1}^{2}+\left(l_{1}+l_{2}\right) \tau_{m, t-1}+p_{u}=0
$$

where $p_{u}=l_{1} l_{2}-g_{u, 1} g_{u, 2} /\left(1-\varepsilon_{\mathrm{OP}}\right)$. Moreover, if Equation (36) has a positive real solution, we have

$$
\tau_{m, t-1}^{*}=\frac{-1\left(l_{1}+l_{2}\right)+\sqrt{\left(l_{1}+l_{2}\right)^{2}-4 p_{u}}}{2}
$$

Proposition 3. Equation (35) cannot have two distinct positive real solutions. 
Proof. Firstly, we consider the following function:

$$
f\left(\tau_{m, t-1}\right)=\prod_{n=1}^{N}\left(\tau_{m, t-1} l_{n}\right)-\frac{1}{1-\varepsilon_{\mathrm{OP}}} \prod_{n=1}^{N} g_{u, n} .
$$

Assume that there exist at least two distinct positive real solutions, e.g., $\tau_{1}$ and $\tau_{2}, 0<\tau_{1}<\tau_{2}$; we then have

$$
f\left(\tau_{1}\right)=f\left(\tau_{2}\right)=0 .
$$

Differentiating $f\left(\tau_{m, t-1}\right)$ with respect to $\tau_{m, t-1}$ yields

$$
\frac{\partial f\left(\tau_{m, t-1}\right)}{\partial \tau_{m, t-1}}=\sum_{n=1}^{N} \prod_{v=1, v \neq n}^{N}\left(\tau_{m, t-1} l_{v}\right) .
$$

Since $\partial f\left(\tau_{m, t-1}\right) / \partial \tau_{m, t-1}>0$ when $\tau_{m, t-1}>0, f\left(\tau_{m, t-1}\right)$ increases in interval $[0,+\infty)$. This means that $f\left(\tau_{1}\right)<f\left(\tau_{2}\right)$, and hence, we cannot obtain (39).

Corollary 4. Assume that Equation (35) has one positive real solution; the transmit power $P_{R m, t-1}$ is lower with higher QoS of the primary network (or lower value of $\varepsilon_{O P}$ ).

Proof. Let us denote $\tau_{1}$ as the positive real solution of (35), we have $\prod_{n=1}^{N}\left(\tau_{1}+l_{n}\right)=1 / 1-\varepsilon_{\mathrm{OP}} \prod_{n=1}^{N} g_{u, n}$. Therefore, it is straightforward that for the fixed values of $l_{n}$ and $g_{u, n}$, if $\varepsilon_{\mathrm{OP}}$ decreases, then $\tau_{1}\left(\right.$ or $\left.P_{\mathrm{R} m, t-1}\right)$ also decreases.

Proposition 5. Equation (35) has one positive real solution when

$$
\prod_{n=1}^{N} l_{n}-\frac{1}{1-\varepsilon_{O P}} \prod_{n=1}^{N} g_{u, n}<0
$$

Proof. As proved in Proposition 3, $f\left(\tau_{m, t-1}\right)$ is a continuous and increasing function in $[0,+\infty)$. Therefore, it is obvious that if $f(0)<0$, Equation (35) always has one real positive solution. Using (38), we obtain (41) and finish the proof.

For example, with $N=2$, the condition in (41) can be written as

$$
l_{1} l_{2}<\frac{g_{u, 1} g_{u, 2}}{1-\varepsilon_{\mathrm{OP}}} \Leftrightarrow p_{u}<0 .
$$

Proposition 6. If Equation (35) has one positive real solution, at high $P_{P T}$ values (i.e., $P_{P T} \longrightarrow+\infty$ ), $P_{R m, t-1}$ is a linear function of $P_{P T}$.

Proof. As $P_{\mathrm{PT}} \longrightarrow+\infty$, we have

$$
g_{u, n} \stackrel{P_{\mathrm{PT}} \rightarrow+\infty}{\approx} \underbrace{\left[\prod_{u=n+1}^{N} \frac{\lambda_{\mathrm{PT}_{u}, \mathrm{PR}}}{\lambda_{\mathrm{PT}_{u}, \mathrm{PR}}+a_{u, n} \lambda_{\mathrm{PT}_{n}, \mathrm{PR}}}\right] \frac{\lambda_{\mathrm{R}_{m, t-1}, \mathrm{PR}}}{b_{n} \lambda_{\mathrm{PT}_{n}, \mathrm{PR}}}}_{g_{u, n}^{*}} .
$$

Therefore, at high $P_{\mathrm{PT}}$ values, Equation (35) becomes

$$
\prod_{n=1}^{N}\left(\tau_{m, t-1}+l_{n}\right)=\frac{1}{1-\varepsilon_{\mathrm{OP}}} \prod_{n=1}^{N} g_{u, n}^{*}
$$

If $\tau_{m, t-1}^{* *}$ is a positive real solution of (44), $\tau_{m, t-1}^{* *}$ does not depend on $P_{\mathrm{PT}}$. Hence, $P_{\mathrm{R} m, t-1}$ becomes a linear function of $P_{\mathrm{PT}}$, i.e., $P_{\mathrm{R}_{m, t-1}} \approx P_{\mathrm{PT} \rightarrow+\infty} \tau_{m, t-1}^{* *} P_{\mathrm{PT}}$.

Remark 7. Before the secondary source starts the data transmission, the best path is chosen by the source, and all the secondary transmitters on the selected path have to adjust their transmit power. In practice, the value of $P_{\mathrm{R} m, t-1}$ can be calculated by the primary network and is then sent to the secondary network. In the conventional UCR schemes (see [45, 46]), the secondary transmitters have to adjust their transmit power, following the instantaneous CSIs between themselves and the primary receivers. However, these methods are very complex due to requirement of perfect CSI estimation and frequent change of the link channels.

3.3. Outage Probability of Secondary Network. This subsection evaluates the e2e OP of M-DF-P and M-AF-P, which can be defined as

$$
\mathrm{OP}_{Z}^{\mathrm{e} 2 \mathrm{e}}=\operatorname{Pr}\left(C_{b}^{\mathrm{e} e \mathrm{e}}<C_{\mathrm{S}, \mathrm{th}}\right)=\prod_{m-1}^{M} \mathrm{OP}_{m, \mathrm{Z}}
$$

where $Z \in\{A F, D F\}, C_{\mathrm{S}, \text { th }}$ is a predetermined target rate of the secondary network, and $\mathrm{OP}_{m, Z}=\operatorname{Pr}\left(C_{m}^{\mathrm{e} 2 \mathrm{e}}<C_{\mathrm{S} \text {,th }}\right)$ is the e2e OP of the $m$ th path. In the following, we attempt to calculate $\mathrm{OP}_{m, \mathrm{Z}}$.

(1) M-DF-P: in this protocol, the e2e OP of the $m t h$ path is calculated as follows:

(1) M-AF-P: because it is too difficult to find an exact expression of $\mathrm{OP}_{m, \mathrm{AF}}$, we attempt to obtain a lowerbound one.

Proposition 8. $O P_{m, D F}$ can be expressed by an exact closedform expression as

$$
O P_{m, D F}=\left\{\begin{array}{l}
1, \text { if } 1-\kappa_{s s}^{2} \theta_{S, m} \leq 0 \\
1-\prod_{t=1}^{L_{m+1}+1}\left[\prod_{n=1}^{N} \frac{\lambda_{P T_{n}, R_{m, t}}}{\lambda_{P T_{m}, R_{m, t}}+\lambda_{R_{m, t-1}, R_{m, t}} \rho_{1, n}}\right] \exp \left(-\lambda_{R_{m, t-1}, R_{m, t}} \rho_{2}\right), 1-\kappa_{s, s}^{2} \theta_{S, m},
\end{array}\right.
$$


where

$$
\begin{aligned}
\theta_{S, m} & =2^{\left(L_{m}+1\right) C_{S, t h}-1, \rho_{1, n}}=\frac{\left(1+\kappa_{p s}^{2}\right) \theta_{S, m} \beta_{n} P_{P T}}{\left(1-\theta_{S, m} \kappa_{s s}^{2}\right) P_{R_{m, t-1}}}, \rho_{2} \\
& =\frac{\sigma_{0}^{2} \theta_{S, m}}{\left(1-\theta_{S, m} \kappa_{s S}^{2}\right) P_{R_{m, t-1}}} .
\end{aligned}
$$

Proof. See Appendix A.

Corollary 9. If $1-\theta_{S, m} \kappa_{s s}^{2}>0, O P_{m, D F}$ at high $P_{P T}$ values can be approximated as

$$
O P_{m, D F} \stackrel{P_{P T} \rightarrow+\infty}{\approx} \prod_{t=1}^{L_{m}+1} \prod_{n=1}^{N} \frac{\lambda_{P T_{n}, P R}}{\lambda_{P T_{n}, P R}+\lambda_{R_{m, t-1}, R_{m, t}} P_{l, n}^{*}},
$$

where

$$
\rho_{1, n}^{*}=\frac{\left(1+\kappa_{p s}^{2} \theta_{S, m} \beta_{n}\right)}{\left(1-\theta_{S, m} \kappa_{s s}^{2}\right) \tau_{m, t-1}^{* *}} .
$$

Proof. Substituting $P_{\mathrm{R}_{m, t-1}} \approx^{P_{\mathrm{PT}} \rightarrow+\infty} \tau_{m, t-1}^{* *} P_{\mathrm{PT}}$ and $\exp (-$ $\left.\lambda_{\mathrm{R}_{m, t-1}, \mathrm{R}_{m, t}} \rho_{2}\right) \approx^{P_{\mathrm{PT}} \rightarrow+\infty} 1$ into (46), we obtain (48).

Similar to [31], we consider two RVs: $X_{1, m}=\min _{q=1, \cdots, T_{1}}($ $\left.\lambda_{\mathrm{R}_{m, q-1}, \mathrm{R}_{m, q}}\right)$ and $X_{2, m}=\min _{r=T_{1}+1, \cdots, L_{m}+1}\left(\lambda_{\mathrm{R}_{m, r-1}, \mathrm{R}_{m, r}}\right)$, where $T_{1, m}=$ $\left\lfloor\left(L_{m}+1\right) / 2\right\rfloor$ and $\lfloor x\rfloor$ is the highest integer that is smaller or equal to $x$. Then, the e2e SINR $\Psi_{\mathrm{R} m, L m+1}$ (see (21) or (22)) can be bounded as in [31]:

$$
\Psi_{\mathrm{R}_{m, L_{m}+1}} \leq \frac{X_{1, m} X_{2, m}}{X_{1, m}+X_{2, m}+1}=\Phi_{m}
$$

Our next objective is to find the distributions of $X_{1, m}$ and $X_{2, m}$ which are presented in Proposition 10 .

Proposition 10. CDF of $X_{1, m}$ and $X_{2, m}$ can be given, respectively, as follows:

$$
F_{X_{1, m}}(x)=\left\{\begin{array}{l}
1,1-\kappa_{s s}^{2} x \leq 0 \\
1-\exp \left(-\rho_{4} \vartheta(x)\right) \sum_{q=1}^{T_{1}} \sum_{n=1}^{N} \frac{\varepsilon_{q, n}}{\vartheta(x)+\chi_{q, n}}, 1-\kappa_{s s}^{2} x>0,
\end{array}\right.
$$

$$
F_{X_{2, m}}(x)=\left\{\begin{array}{l}
1,1-\kappa_{s s}^{2} x \leq 0 \\
1-\exp \left(-\rho_{6} \vartheta(x)\right) \sum_{r=T_{1}+1}^{L_{m}+1} \sum_{l=1}^{N} \frac{\varepsilon_{r, l}}{\vartheta(x)+\omega_{r, l}}, \quad 1-\kappa_{s s}^{2} x>0,
\end{array}\right.
$$

where

$$
\begin{aligned}
& \rho_{3, n}=\frac{\beta_{n}\left(1+\kappa_{p s}^{2}\right) P_{P T}}{P_{R_{m, q-1}}}, \rho_{4}=\sum_{q=1}^{T_{1}} \frac{\lambda_{R_{m, q-1}, R_{m, q}} \sigma_{0}^{2}}{P_{R_{m, q-1}}}, \vartheta(x)=\frac{x}{1-\kappa_{s s}^{2} x} \\
& \chi_{q, n}=\frac{\lambda_{P T_{n}, R_{m, q}}}{\lambda_{R_{m, q-1}, R_{m, q}} \rho_{3, n}}, \varepsilon_{q, n}=\chi_{q, n} \prod_{a=1}^{T_{1}} \prod_{\substack{b=1 \\
(a, b) \neq(q, n)}}^{N} \frac{\chi_{a, b}}{\chi_{a, b}-\chi_{q, n}}, \\
& \rho_{5, l}=\frac{\beta_{l}\left(1+\kappa_{p s}^{2}\right) P_{P T}}{P_{R_{m, r-1}}}, \rho_{6}=\sum_{r=T_{1}+1}^{L_{m}+1} \frac{\lambda_{R_{m, r-1}, R_{m, r}} \sigma_{0}^{2}}{P_{R_{m, r-1}}}, \omega_{r, l} \\
& =\frac{\lambda_{P T_{l}, R_{m, r}}}{\lambda_{R_{m, r-1}, R_{m, r}} \rho_{5, l}}, \xi_{r, l}=\omega_{r, l} \prod_{a=T_{1}+1}^{L_{m}+1} \prod_{\substack{b=1 \\
(a, b) \neq(r, l)}}^{N} \frac{\omega_{a, b}}{\omega_{a, b}-\omega_{r, l}} .
\end{aligned}
$$

Proof. See Appendix B.

From (52), if $1-\kappa_{s s}^{2} x \leq 0, f_{X_{2, m}}(x)=0$, and if $1-\kappa_{s s}^{2} x>0$, PDF of $X_{2, m}$ can be obtained as

$$
\begin{aligned}
f_{X_{2, m}}(x)= & \sum_{r=T_{1}+1}^{L_{m}+1} \sum_{l=1}^{N} \frac{\xi_{r, l}}{\left(\vartheta(x)+\omega_{r, l}\right)^{2}\left(1-\kappa_{s s}^{2} x\right)^{2}} \exp \left(-\rho_{6} \vartheta(x)\right) \\
& +\sum_{r=T_{1}+1}^{L_{m}+1} \sum_{l=1}^{N} \frac{\xi_{r, l} \rho_{6}}{\left(\vartheta(x)+\omega_{r, l}\right)^{2}\left(1-\kappa_{s s}^{2} x\right)^{2}} \exp \left(-\rho_{6} \vartheta(x)\right) .
\end{aligned}
$$

Next, we derive the lower-bound expression of $\mathrm{OP}_{m, \mathrm{AF}}$ as given in Proposition 11.

Proposition 11. If $1-\left(k_{S S}^{4}+2 k_{S S}^{2}\right) \theta_{S, m \leq 0}$, then $O P_{m, A F}=1$, and if $1-\left(k_{S S}^{4}+2 k_{S S}^{2}\right) \theta_{S, m>0}, O P m_{A F}$ is bounded by

$$
O P_{m, A F \geq 1-} \int_{v_{1}}^{v_{2}}\left[1-F_{X_{1, m}}\left(\frac{\theta_{S, m} x+\theta_{S, m}}{x-\theta_{S, m}}\right)\right] f_{X_{2, m}}(x) d x
$$

where

$$
v_{1}=\frac{\left(1+k_{S S}^{2}\right) \theta_{S, m}}{1+k_{S S}^{2} \theta_{S, m}}, v_{2}=\frac{1}{k_{S S}^{2}}
$$

Proof. See Appendix C.

Remark 12. Firstly, $\mathrm{OP}_{m, \mathrm{AF}}^{\mathrm{LB}}$ can be numerically calculated by using MATLAB or Mathematica. Secondly, as $P_{\mathrm{PT}} \rightarrow+\infty$, the e2e OP of M-DF-P and M-AF-P does not depend on $P_{\mathrm{PT}}$. Indeed, $\gamma_{\mathrm{R}_{m, t-1}}, \mathrm{R}_{m, t}$ in (12) can be approximated as 


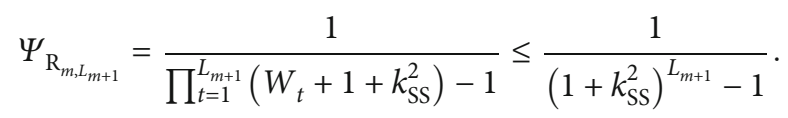

We can observe from (58) that $\gamma_{\mathrm{R}_{m, t-1}}, \mathrm{R}_{m, t}$ at high $P_{\mathrm{PT}}$ values do not depend on $P_{\mathrm{PT}}$, and it is the reason why $\mathrm{OP}_{m, Z}\left(\mathrm{OP}_{Z}^{\mathrm{e} e \mathrm{e}}\right)$ converges to a constant as $P_{\mathrm{PT}} \rightarrow+\infty S$. This also means that the diversity order of both M-DF-P and MAF-P is zero.

Corollary 13. $O P_{m, A F}$ always equals to 1 if $1-\left(\left(1+k_{S S}^{2}\right)^{L_{m}+1}\right.$ $-1) \theta_{S, m} \leq 0$.

Proof. At first, we can rewrite the e2e SINR $\Psi_{\mathrm{R}_{m, L_{m+1}}}$ under the following form:

$$
\Psi_{\mathrm{R}_{m, L_{m+1}}}=\frac{1}{\prod_{t=1}^{L_{m+1}}\left(1+1 / \gamma R_{m, t-1}, R_{m, t}\right)-1} .
$$

Combining (12) and (59), it is straightforward that

$$
\Psi_{\mathrm{R}_{m, L m+1}}=\frac{1}{\prod_{t=1}^{L_{m}+1}\left(W_{t}+1+\kappa_{s s}^{2}-1\right)} \leq \frac{1}{\left(1+\kappa_{s s}^{2}\right)^{L_{m}+1}-1},
$$

where

$$
W_{t}=\frac{\left(1+\kappa_{\mathrm{ps}}^{2}\right) \sum_{n=1}^{N} \beta_{n} P_{\mathrm{PT}_{\mathrm{T}}} \varphi_{\mathrm{PT}_{n} \mathrm{R}_{m, t}}+\sigma_{0}^{2}}{P_{\mathrm{R}_{m, t-1}} \varphi_{\mathrm{R}_{m, t-1}, \mathrm{R}_{m, t}}} .
$$

Therefore, if $1-\left(\left(1+\kappa_{\mathrm{ss}}^{2}\right)^{L_{m}+1}-1\right) \theta_{\mathrm{S}, m} \leq 0$, then $\mathrm{OP}_{m, \mathrm{AF}}$ $=1$.

Remark 14. From Proposition 8, we can see that if $\kappa_{s s}^{2} \geq$ $\left(\kappa_{\mathrm{ss}}^{\mathrm{DF}}\right)^{2}=\max _{m=1,2, \cdots, M}\left(1 / \theta_{\mathrm{S}, m}\right)$, all of the paths in M-DF-P are in outage, and hence, M-DF-P is always in outage, regardless of the values of the other system parameters. For the AF method, from Corollary 13, M-AF-P is always in outage if

$$
\kappa_{\mathrm{ss}}^{2} \geq\left(\kappa_{\mathrm{sS}}^{\mathrm{AF}}\right)^{2}=\max _{m=1,2, \cdots, M}\left(\left(\frac{1+\theta_{\mathrm{S}, m}}{\theta_{\mathrm{S}, m}}\right)^{1 / L_{m}+1}-1\right),
$$

where $\left(\kappa_{\mathrm{ss}}^{\mathrm{DF}}\right)^{2}$ and $\left(\kappa_{\mathrm{ss}}^{\mathrm{AF}}\right)^{2}$ are called as limited hardware impairment levels of M-DF-P and M-AF-P, respectively. Because $\left(\kappa_{\mathrm{ss}}^{\mathrm{DF}}\right)^{2} \geq\left(\kappa_{\mathrm{ss}}^{\mathrm{AF}}\right)^{2}, \mathrm{M}$-AF-P is more sensitive to $\mathrm{HI}$ than M-DF-P.

Proposition 15. When the transceiver hardware is perfect, i.e., $\kappa_{s s}^{2}=\kappa_{s p}^{2}=\kappa_{p s}^{2}=0$, we obtain a lower-bound closed-form expression of $O P_{m, A F}$ by
TABLE 2: Values of the system parameters.

\begin{tabular}{lc}
\hline System parameters & Values \\
\hline$\sigma_{0}^{2}$ & 1 \\
$P L$ & 3 \\
$C P$, th & 0.2 \\
$\varepsilon \mathrm{OP}$ & 0.05 \\
\hline
\end{tabular}

$O P_{m, A F} \geq 1-\sum_{q=1}^{T_{1}} \sum_{n=1}^{N} \sum_{r=T_{1}+1}^{L_{m}+1} \sum_{l=1}^{N}\left[\frac{\mu_{1}}{\mu_{3}-\mu_{2}}+\frac{\mu_{1} \mu_{2}}{\mu_{2}-\mu_{3}} \log _{e}\left(\frac{\mu_{2}}{\mu_{3}}\right)\right]$,

where

$$
\begin{aligned}
& \chi_{q, n}^{*}=\frac{\lambda_{P T_{n}, R_{m, q-1}}}{\lambda_{R_{m, q-1}, R_{m, q}} \beta_{n} P_{P T}}, \varepsilon_{q, n}^{*}=\chi_{q, n}^{*} \prod_{a=1}^{T_{1}} \prod_{\substack{b=1 \\
(a, b) \neq(q, n)}}^{N} \frac{\chi_{a, b}^{*}}{\chi_{a, b}^{*}-\chi_{q, n}^{*}}, \\
& \omega_{r, l}^{*}=\frac{\lambda_{P T_{l}, R_{m, r}} P_{R_{m, r-1}}}{\lambda_{R_{m, r-1}, R_{m, r}} \beta_{l} P_{P T}}, \varepsilon_{r, l}^{*}=\omega_{r, l}^{*} \prod_{a=T_{1}+1}^{L_{m}+1} \prod_{\substack{b=1 \\
(a, b) \neq(r, l)}}^{N} \frac{\omega_{a, b}^{*}}{\omega_{a, b}^{*}-\omega_{r, l}^{*}}, \\
& \mu_{1}=\frac{\varepsilon_{q, n}^{*} \xi_{r, l}^{*}}{\theta_{S, m}+\chi_{q, n}^{*}}, \mu_{2}=\frac{\theta_{S, m}^{2}+\theta_{S, m}}{\theta_{S, m}+\chi_{q, n}^{*}}, \mu_{3}=\theta_{S, m}+\omega_{r, l}^{*} .
\end{aligned}
$$

Proof. See Appendix D.

\section{Numerical Results}

This section provides the Monte-Carlo-based computer simulations to verify the analysis as well as to compare the e2e $\mathrm{OP}$ of M-DF-P and M-AF-P. Simulation environment is a two-dimensional plane Oxy, in which all the primary and secondary nodes are placed. We assume that there are two primary transmitters, and their coordinates are $\mathrm{PT}_{1}(0.35$, $0.5)$ and $\mathrm{PT}_{2}(0.6,0.5)$, while $\mathrm{PR}$ is located at $(0.45,0.5)$. As mentioned in "System Model," the transmit power of $\mathrm{PT}_{1}$ is higher than that of $\mathrm{PT}_{2}$; hence, we can set $\beta_{1}=1$ and $\beta_{2}=$ 0.2 . In the secondary network, assume that there are 03 available paths $(M=3)$, and the number of relays on the paths is $L_{1}=1, L_{2}=2$, and $L_{3}=3$. In addition, the source and destination nodes are located at $(0,0)$ and $(0,1)$, respectively, and the relay node $R_{m, t}$ is placed at $\left(\left(t / L_{m}+1\right), 0\right)$, where $m=1,2,3$ and $t=1, \cdots, L_{m}$. To focus on performance trend and performance comparison between M-DF-P and M-AF-P, some system parameters are fixed in all the simulations, as given in Table 2.

Remark 16. Although we do not investigate the impact of the number of paths $(M)$ and the number of the primary transmitters $(N)$ on the e2e OP of the secondary networks, we can guess that the performance of M-DF-P and M-AF-P is 


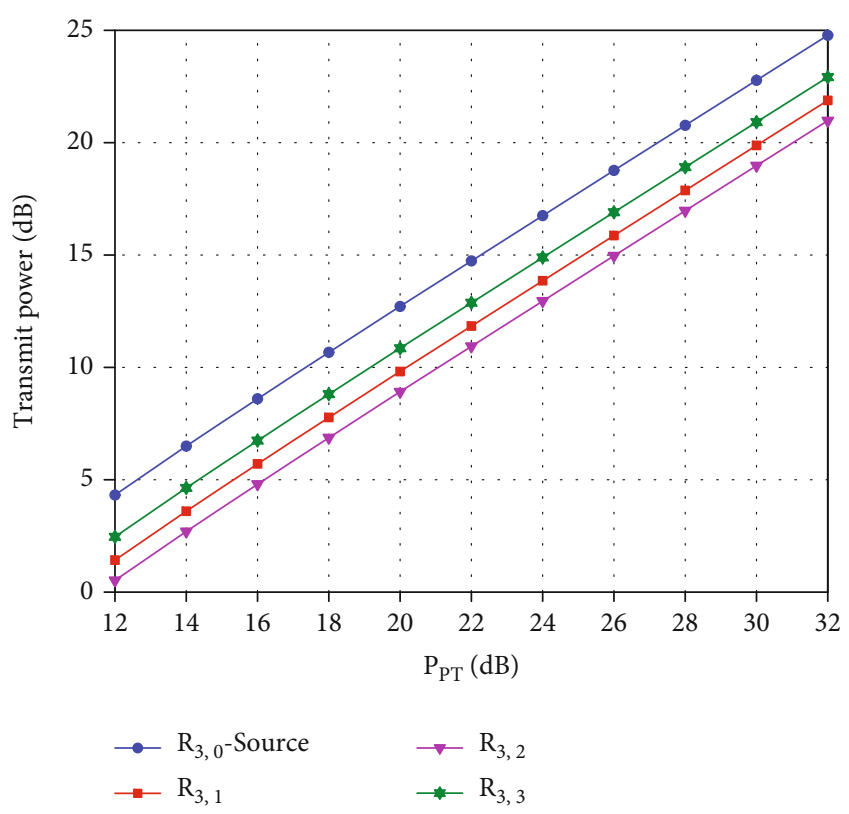

FIgURE 2: Transmit power of the secondary transmitters on the third path $\left(L_{3}=3\right)$ as a function of $P_{\mathrm{PT}}$ in $\mathrm{dB}$ when $\kappa_{s p}^{2}=0.01$.
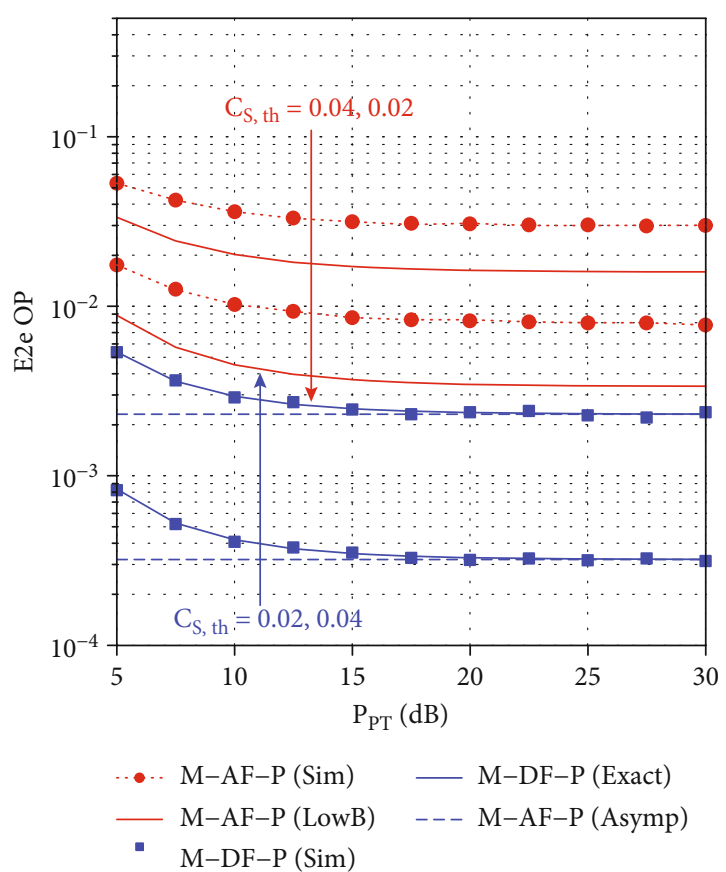

FIGURE 3: End-to-end outage probability of M-DF-P and M-AF-P as a function of $P_{\mathrm{PT}}$ in $\mathrm{dB}$ when $\kappa_{s s}^{2}=0.05, \kappa_{s p}^{2}=\kappa_{p s}^{2}=0.01$.

better with a higher value of $M$ and a lower value of $N$. Also, we can expect that the performance of M-DF-P and M-AF-P is better with higher value of $\varepsilon_{O P}$, and vice verse.

In Figure 2, we assume that the third path is selected and presents the transmit power of $\mathrm{R}_{3,0}$ (Source), $\mathrm{R}_{3,1}, \mathrm{R}_{3,2}$, and $\mathrm{R}_{3,3}$ as a function of $P_{\mathrm{PT}}$ in $\mathrm{dB}$. As seen from Figure 2, the transmit power of all the secondary transmitters is higher than zero when $P_{\mathrm{PT}}$ changes from $12 \mathrm{~dB}$ to $32 \mathrm{~dB}$. This means

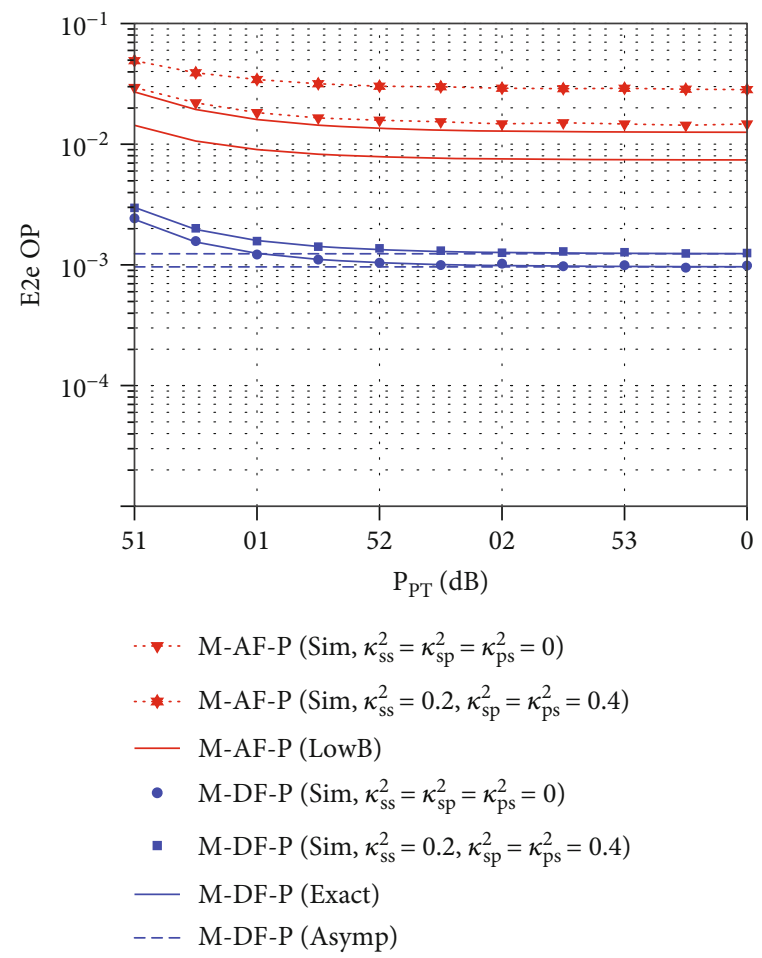

FIGURE 4: End-to-end outage probability of M-DF-P and M-AF-P as a function of $P_{\mathrm{PT}}$ in $\mathrm{dB}$ when $C_{S, \text { th }}=0.03$.

that the secondary transmitters are allowed to access the licensed band to transmit the data. As proved in Proposition 6 , we also observe that the transmit power linearly increases with the increase of $P_{\mathrm{PT}}$. Also in this figure, the transmit power of $R_{3,0}$ is highest, and that of $R_{3,2}$ is lowest. It is due to the fact that the distance between $R_{3,0}$ and $P R$ is furthest, and $R_{3,2}$ is nearest to $P R$.

Figure 3 compares the performance of M-DF-P and MAF-P when $\kappa^{2}{ }_{s s}=0.05$ and $\kappa^{2}{ }_{\text {sp }}=\kappa_{\text {ps }}^{2}=0.01$. We can see that the performance of M-DF-P is better than that of M-AF-P for all values of $P_{\mathrm{PT}}$. As mentioned in Remark 12, the e2e OP of M-DF-P and M-AF-P in Figure 3 converge to a constant when $P_{\mathrm{PT}}$ is high enough. In addition, the performance of the proposed protocols is better as $C_{\mathrm{S} \text {,th }}$ decreases. It is worth noting that for M-DF-P, the simulation results (denoted by Sim) verify the theoretical ones, where Exact denotes the theoretical results obtained by using (46) and Asymp denotes the approximate expression of the e2e OP at high $P_{\mathrm{PT}}$ regimes (see (48)). For $\mathrm{M}-\mathrm{AF}-\mathrm{P}$, there exists gap between the simulation results (Sim) and the theoretical results (LowB) because the usage of (56) for evaluating the outage performance.

In Figure 4, the e2e OP of M-DF-P and M-AF-P presents as a function of $P_{\mathrm{PT}}$ in $\mathrm{dB}$ when $C_{\mathrm{S} \text {,th }}=0.03$. In this figure, we consider two cases: the transceiver hardware is perfect $\left(\kappa_{\mathrm{ss}}^{2}\right.$ $\left.=\kappa_{\mathrm{sp}}^{2}=\kappa_{\mathrm{ps}}^{2}=0\right)$ and imperfect $\left(\kappa_{\mathrm{ss}}^{2}=\kappa_{\mathrm{sp}}^{2}=\kappa_{\mathrm{ps}}^{2}=0.04\right)$.

As expected, the outage performance of the proposed protocols with perfect transceiver hardware is better. Similar to Figure 3, M-DF-P outperforms M-AF-P, and the OP curves rapidly converge to constants. It is worth noting that 


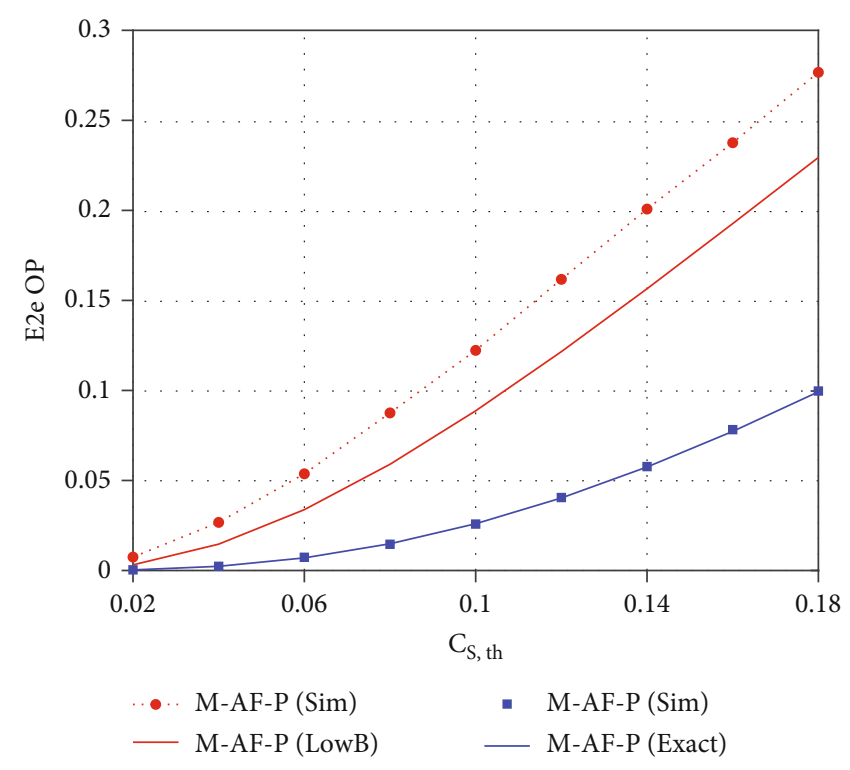

Figure 5: End-to-end outage probability of M-DF-P and M-AF-P as a function of $C_{S, \text { th }}$ when $P_{\mathrm{PT}}=15 \mathrm{~dB}, \kappa_{\mathrm{ss}}^{2}=\kappa_{\mathrm{sp}}^{2}=\kappa_{\mathrm{ps}}^{2}=0$.

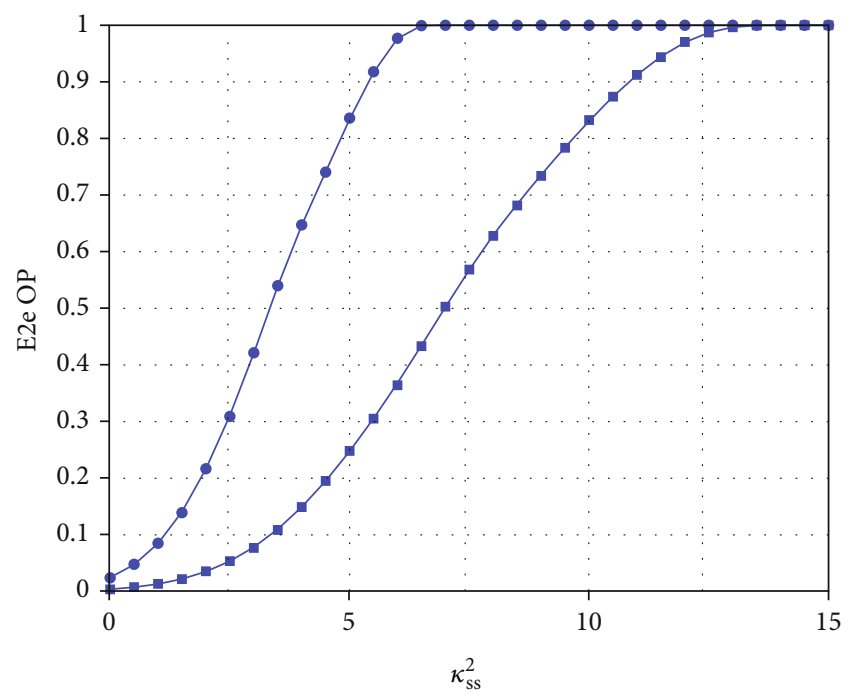

- $\quad \mathrm{M}-\mathrm{DF}-\mathrm{P}\left(\mathrm{Sim}, \mathrm{C}_{\mathrm{S}, \text { th }}=0.05\right)$

- $\quad M-D F-P\left(\operatorname{Sim}, C_{S, \text { th }}=0.1\right)$

- M-DF-P (Exact)

Figure 6: End-to-end outage probability of M-DF-P as a function of $\kappa_{\mathrm{ss}}^{2}$ when $P_{\mathrm{PT}}=30 \mathrm{~dB}, \kappa_{\mathrm{sp}}^{2}=\kappa_{\mathrm{ps}}^{2}=\kappa_{\mathrm{ss}}^{2} / 5$.

when the hardware impairments are relaxed, the closed-form expression in (63) is used to calculate the lower-bound e2e OP for M-AF-P, instead of (56).

Figure 5 compares the performance of M-DF-P and MAF-P with different values of $C_{S \text {,th }}$. In this figure, the perfect transceiver hardware is assumed, and the transmit power $P_{\mathrm{PT}}$ is fixed by $15 \mathrm{~dB}$. We can see from Figure 5 that the e $2 \mathrm{e}$ $\mathrm{OP}$ values of the proposed protocols increase as $C_{\mathrm{S} \text {,th }}$ increases. Moreover, the performance of M-DF-P is better

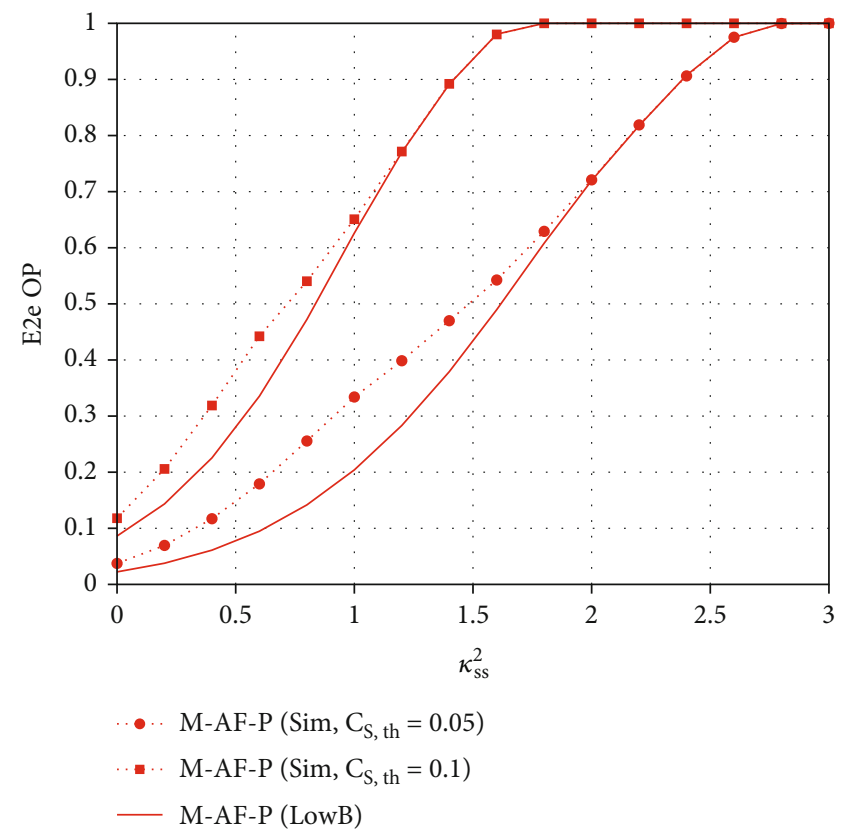

FIGURE 7: End-to-end outage probability of M-AF-P as a function of $\kappa_{\mathrm{ss}}^{2}$ when $P_{\mathrm{PT}}=30 \mathrm{~dB}, \kappa_{\mathrm{sp}}^{2}=\kappa_{\mathrm{ps}}^{2}=\kappa_{\mathrm{ss}}^{2} / 5$.

than that of M-AF-P, and the performance gap is higher as $C_{S \text {,th }}$ increases.

In Figure 6, we present the outage performance of M-DF$\mathrm{P}$ as a function of $\kappa_{\mathrm{ss}}^{2}$ when $P_{\mathrm{PT}}=30 \mathrm{~dB}$, and $\kappa_{\mathrm{sp}}^{2}=\kappa_{\mathrm{ps}}^{2}=\kappa_{\mathrm{ss}}^{2} / 5$.

Figure 6 illustrates that the e2e OP rapidly increases as $\kappa_{\mathrm{ss}}^{2}$ increases. We also see that when the HI level is high enough, M-DF-P is always in outage. As given in Remark 14, the limited hardware impairment levels of M-DF-P in this figure can be obtained as

$$
\left(\kappa_{\mathrm{ss}}^{\mathrm{DF}}\right)^{2}=\left\{\begin{array}{l}
13.93, C_{\mathrm{S}, \text { th }}=0.05 \\
6.72, C_{\mathrm{S}, \mathrm{th}}=0.1
\end{array}\right.
$$

Figure 7 depicts the performance of M-AF-P as a function of $\kappa_{\text {ss }}^{2}$ when $P_{\mathrm{PT}}=30 \mathrm{~dB}$, and $\kappa_{\mathrm{sp}}^{2}=\kappa_{\mathrm{ps}}^{2}=\kappa_{\mathrm{ss}}^{2} / 5$. Similar to M-DF-P in Figure 6, the performance of M-AF-P is worse when $\kappa_{\text {ss }}^{2}$ increases. Also, M-AF-P is always in outage if $\kappa_{\text {ss }}^{2}$ $\geq\left(\kappa_{\mathrm{ss}}^{\mathrm{AF}}\right)$, where $\left(\kappa_{\mathrm{ss}}^{\mathrm{AF}}\right)^{2}$ is calculated by using $(62)$ :

$$
\left(\kappa_{\mathrm{ss}}^{\mathrm{AF}}\right)^{2}=\left\{\begin{array}{l}
2.86, C_{\mathrm{S}, \mathrm{th}}=0.05 \\
1.77, C_{\mathrm{S}, \mathrm{th}}=0.1
\end{array}\right.
$$

From Figures 3-7, we can see that M-DF-P not only outperforms M-AF-P but also obtains much higher value of the limited HI level.

\section{Conclusion}

This paper proposed, evaluated, and compared the e2e OP of M-DF-P and M-AF-P in the UCR networks. The interesting results obtained in this paper can be summarized as follows: (i) the transmit power of the secondary transmitters can 
linearly increase with the transmit power of the primary transmitters, (ii) the OP performance of M-DF-P is significantly better than that of M-AF-P, (iii) there exist error floors that means the diversity gain of M-DF-P and M-AF-P equals to zero, and (iv) M-AF-P is more sensitive to the HI than MDF-P, i.e., the limited HI level of M-AF-P is much lower than that of M-DF-P. Finally, the OP performance for M-DF-P and M-AF-P can be enhanced by increasing the number of paths between the secondary source and destination and by equipping better transceiver hardware for the secondary nodes (i.e., lower hardware impairment level).

\section{Appendix}

\section{A. Proof of Proposition 8}

Using (13), we have

$$
\begin{aligned}
\mathrm{OP}_{m . \mathrm{DF}} & =\operatorname{Pr}\left(\min _{t=1,2, \cdots, L_{m}+1}\left(\gamma_{\mathrm{R}_{m, t-1} \mathrm{R} m, t}\right)<\theta_{\mathrm{S}, m}\right) \\
& =1-\prod_{t=1}^{L_{m}+1}\left(1-\operatorname{Pr}\left(\gamma_{\mathrm{R}_{m, t-1} \mathrm{R} m, t}<\theta_{\mathrm{S}, m}\right)\right)
\end{aligned}
$$

where $\theta_{\mathrm{S}, m}$ is given by (47).

Combining (12) and (A.1) yields

$$
\begin{aligned}
\operatorname{Pr} \gamma_{\mathrm{R}_{m, t-1} \mathrm{R} m, t}<\theta_{\mathrm{S}, m} & =\operatorname{Pr}\left(\left(1-\kappa_{\mathrm{ss}}^{2} \theta_{\mathrm{S}, m}\right) P_{\mathrm{R}_{m, t-1}} \varphi_{\mathrm{R}_{m, t-1}, \mathrm{R}_{m, t}}\right. \\
& \left.<\left(1+\kappa_{\mathrm{ps}}^{2}\right) \theta_{\mathrm{S}, m} \sum_{n=1}^{N} \beta_{n} P_{\mathrm{PT}_{1}} \varphi_{\mathrm{PT}_{n}, \mathrm{R}_{m, t}}+\sigma_{0}^{2} \theta_{\mathrm{S}, m}\right) .
\end{aligned}
$$

We can see from (A.2) that if $1-\theta_{\mathrm{S}, m} \kappa_{\mathrm{ss}}^{2} \leq 0, \operatorname{Pr}($ $\left.\gamma_{\mathrm{R}_{m, t-1} \mathrm{R} m, t}<\theta_{\mathrm{S}, m}\right)=1$, and if $\theta_{\mathrm{S}, m} \kappa_{\mathrm{ss}}^{2}>0$, we have

$$
\begin{aligned}
\operatorname{Pr}\left(\gamma_{\mathrm{R}_{m, t-1}, \mathrm{R}_{m, t}}<\theta_{\mathrm{S}, m}\right)= & \operatorname{Pr}\left(\varphi_{\mathrm{R}_{m, t-1}, \mathrm{R}_{m, t}}<\rho_{1, n} \sum_{n=1}^{N} \varphi_{\mathrm{PT}_{n}, \mathrm{R}_{m, t}}+\rho_{2}\right) \\
= & \int_{0}^{+\infty} \cdots \int_{0}^{+\infty} F_{\varphi_{\mathrm{R}_{m, t-1}, \mathrm{R}_{m, t}}}\left(\rho_{1, n} \sum_{n=1}^{N} x_{n}+\rho_{2}\right) \\
& \cdot \prod_{n=1}^{N} f_{\varphi_{\mathrm{PT}_{n}, \mathrm{R}_{m, t}}}\left(x_{n}\right) d x_{n},
\end{aligned}
$$

where $\rho_{1, n}$ and $\rho_{2}$ are given by (47).

Substituting CDF and PDF in (1) into (A.3), after some manipulation, we can obtain $\operatorname{Pr}\left(\gamma R_{m, t-1}, R_{m, t}<\theta_{S, m}\right)$. Then, we substitute $\operatorname{Pr}\left(\gamma R_{m, t-1}, R_{m, t}<\theta_{S, m}\right)$ into (A.1) to obtain (46), and the proof is also completed.

\section{B. Proof of Proposition 10}

At first, using (12), CDF of $X_{1, m}$ is formulated as

$$
\begin{aligned}
F_{X_{1, m}}(x)= & 1-\prod_{q=1}^{T_{1}}\left(1-\operatorname{Pr}\left(\gamma_{\mathrm{R}_{m, q-1}, \mathrm{R}_{m, q}}<x\right)\right) \\
= & 1-\prod_{q=1}^{T_{1}}\left[1-\operatorname{Pr}\left(\left(1-\kappa_{\mathrm{ss}}^{2} x\right) P_{\mathrm{R}_{m, q-1}} \varphi_{\mathrm{R}_{m, q-1}, \mathrm{R}_{m, q}}\right.\right. \\
& \left.\left.<\left(1+\kappa_{\mathrm{ps}}^{2}\right) x \sum_{n=1}^{N} \beta_{n} P_{\mathrm{PT}_{1}} \varphi_{\mathrm{PT}_{n}, \mathrm{R}_{m, q}}+\sigma_{0}^{2} x\right)\right] .
\end{aligned}
$$

It can be observed that if $1-\kappa_{\mathrm{ss}}^{2} x \leq 0$, then $F_{X_{1, m}}(x)=1$, and if $1-\kappa_{\mathrm{ss}}^{2} x>0$, we have

$$
\begin{aligned}
F_{X_{1, m}}(x)= & 1-\prod_{q=1}^{T_{1}}\left[1-\int_{0}^{+\infty} \cdots \int_{0}^{+\infty} F_{\varphi_{\mathrm{R}_{m}, q-1}, \mathrm{R}_{m}, q}\right. \\
& \left.\cdot\left(\vartheta(x) \sum_{n=1}^{N} \rho_{3, n} x_{n}+\frac{\sigma_{0}^{2}}{P_{\mathrm{R}_{m, q-1}}} \vartheta(x)\right) \prod_{n=1}^{N} f_{\varphi_{\mathrm{PT}_{n}, \mathrm{R}_{m}, q}}\left(x_{n}\right) d x_{n}\right] \\
= & 1-\exp \left(-\rho_{4} \vartheta(x)\right) \prod_{q=1}^{T_{1}} \prod_{n=1}^{N} \frac{\lambda_{\mathrm{PT}_{n}, \mathrm{R}_{m, q}}}{\lambda_{\mathrm{PT}_{n}, \mathrm{R}_{m, q}}+\lambda_{\mathrm{R}_{m, q-1}, \mathrm{R}_{m, q}} \rho_{3, n} \vartheta(x)},
\end{aligned}
$$

where $\rho_{3, n}, \rho_{4}$ and $\vartheta(x)$ are given as in (53).

Then, $F_{X_{1, m}}(x)$ in (B.2) can be expressed as in (51) if $\chi_{a, b} \neq \chi_{q, n}$ if $(a, b) \neq(q, n)$, where $\chi_{q, n}$ is given by (53).

Next, with the same method deriving $F_{X_{1, m}}(x)$, we can obtain $F_{X_{2, m}}(x)$ as presented in (52), $\omega_{a, b} \neq \omega_{r, l}$ if $(a, b) \neq$ $(r, l)$.

\section{Proof of Proposition 11}

At first, using (50), we have

$$
\mathrm{OP}_{m, \mathrm{AF}}=\operatorname{Pr}\left(\Psi_{\mathrm{R}_{m, L_{m}+1}}<\theta_{\mathrm{S}, m}\right) \geq \underbrace{\operatorname{Pr}\left(\frac{X_{1, m} X_{2, m}}{X_{1, m}+X_{2, m}+1}<\theta_{\mathrm{S}, m}\right)}_{\mathrm{OP}_{m, \mathrm{AF}}^{\mathrm{LB}}} .
$$

Considering $\mathrm{OP}_{m, \mathrm{AF}}^{\mathrm{LB}}$ in (C.1), it can be formulated as

$$
\begin{aligned}
\mathrm{OP}_{m, \mathrm{AF}}^{\mathrm{LB}}= & \operatorname{Pr}\left(X_{1, m}\left(X_{2, m}-\theta_{\mathrm{S}, m}\right)<X_{2, m} \theta_{\mathrm{S}, m}+\theta_{\mathrm{S}, m}\right) \\
= & \operatorname{Pr}\left(X_{2, m}<\theta_{\mathrm{S}, m}\right)+\operatorname{Pr} \\
& \cdot\left(X_{2, m} \geq \theta_{\mathrm{S}, m}, X_{1, m}<\frac{X_{2, m} \theta_{\mathrm{S}, m}+\theta_{\mathrm{S}, m}}{X_{2, m}-\theta_{\mathrm{S}, m}}\right) .
\end{aligned}
$$

It is straightforward that if $1-\left(\kappa_{\mathrm{ss}}^{4}+2 \kappa_{\mathrm{sS}}^{2}\right) \theta_{\mathrm{S}, m} \leq 0$, then

$\mathrm{OP}_{m, \mathrm{AF}}^{\mathrm{LB}}=\operatorname{Pr}\left(X_{2, m}<\theta_{\mathrm{S}, m}\right)+\operatorname{Pr}\left(X_{2, m} \geq \theta_{\mathrm{S}, m}\right)=1$. 
In case that $1-\left(\kappa_{\mathrm{ss}}^{4}+2 \kappa_{\mathrm{ss}}^{2}\right) \theta_{\mathrm{S}, m}>0$, Equation (C.2) can be written as follows:

$$
\begin{aligned}
\mathrm{OP}_{m, \mathrm{AF}}^{\mathrm{LB}}= & F_{X_{2, m}}\left(\frac{\left(1+\kappa_{\mathrm{ss}}^{2}\right) \theta_{\mathrm{S}, m}}{1-\kappa_{\mathrm{ss}}^{2} \theta_{\mathrm{S}, m}}\right)+\int_{\frac{\left(1+\kappa_{s}^{2}\right) \theta_{S, m}}{1-k_{\mathrm{ss}}^{2} \theta_{S, m}}}^{1 / \kappa_{\mathrm{ss}}^{2}} F_{X_{1, m}} \\
& \cdot\left(\frac{\theta_{\mathrm{S}, m} x+\theta_{\mathrm{S}, m}}{x-\theta_{\mathrm{S}, m}}\right) f_{X_{2, m}}(x) d x
\end{aligned}
$$

Combining (57), (C.1), and (C.4) together, we can obtain (56), and Proposition 11 is then proved.

\section{Proof of Proposition 15}

Firstly, using $\kappa_{\mathrm{ss}}^{2}=\kappa_{\mathrm{sp}}^{2}=\kappa_{\mathrm{ps}}^{2}=0$ and (12), we have the following inequality:

$$
\begin{aligned}
\gamma_{\mathrm{R}_{m, t-1}, \mathrm{R}_{m, t}} & =\frac{P_{\mathrm{R}_{m, t-1}} \varphi_{\mathrm{R}_{m, t-1}, \mathrm{R}_{m, t}}}{\sum_{n=1}^{N} \beta_{n} P_{\mathrm{PT}_{1}} \varphi_{\mathrm{PT}_{n}, \mathrm{R}_{m, t}}+\sigma_{0}^{2}} \leq \frac{P_{\mathrm{R}_{m, t-1}} \varphi_{\mathrm{R}_{m, t-1}, \mathrm{R}_{m, t}}}{\sum_{n=1}^{N} \beta_{n} P_{\mathrm{PT}} \varphi_{\mathrm{PT}_{n}, \mathrm{R}_{m, t}}} \\
& =\gamma_{\mathrm{R}_{m, t-1}, \mathrm{R}_{m, t}}^{*}
\end{aligned}
$$

Setting $Y_{1, m}=\min _{q=1, \cdots, T_{1}}\left(\gamma_{\mathrm{R}_{m, q-1}, \mathrm{R}_{m, q}}^{*}\right)$ and $Y_{2, m}=\min _{r=T_{1}+1, \cdots, L_{m}+1}$ $\left(\gamma_{\mathrm{R}_{m, r-1}, \mathrm{R}_{m, r}}^{*}\right)$, we have

$$
\mathrm{OP}_{m, \mathrm{AF}} \geq \underbrace{\operatorname{Pr}\left(\frac{Y_{1, m} Y_{2, m}}{Y_{1, m}+Y_{2, m}+1} \leq \theta_{\mathrm{S}, m}\right)}_{\mathrm{OP}_{m, \mathrm{AF}}^{\mathrm{LB}, \mathrm{F}}} .
$$

Similar to the derivation of $\mathrm{OP}_{m, \mathrm{AF}}^{\mathrm{LB}}$ (see (C.1)-(C.4)), we also obtain

$$
\mathrm{OP}_{m, \mathrm{AF}}^{\mathrm{LB}, *}=1-\int_{\theta_{\mathrm{S}, m}}^{+\infty}\left[1-F_{Y_{1, m}}\left(\frac{\theta_{\mathrm{S}, m} x+\theta_{\mathrm{S}, m}}{x-\theta_{\mathrm{S}, m}}\right)\right] f_{Y_{2, m}}(x) d x
$$

Interchanging $\mathrm{RV}$, i.e., $=x-\theta_{\mathrm{S}, m}$, we arrive at

$\mathrm{OP}_{m, \mathrm{AP}}^{\mathrm{LB}, *}=1-\int_{0}^{+\infty}\left[1-F_{Y_{1, m}}\left(\frac{\theta_{\mathrm{S}, m} y+\theta_{\mathrm{S}, m}^{2}+\theta_{\mathrm{S}, m}}{y}\right)\right] f_{Y_{2, m}}\left(y+\theta_{\mathrm{S}, m}\right) d y$.

Similar to the derivation of $F_{X_{1, m}}(x)$ in (51), we have

$$
F_{Y_{1, n}}(x)=1-\sum_{q=1}^{T_{1}} \sum_{n=1}^{N} \frac{\varepsilon_{q, n}^{*}}{x+\chi_{q, n}^{*}},
$$

where $\chi_{q, n}^{*}$ and $\varepsilon_{q, n}^{*}$ are given by (64).
With the same manner as deriving $f_{X_{2, m}}(x)$ in (55), we have

$$
f_{Y_{2, m}}(x)=\sum_{r=T_{1}+1}^{L_{m}+1} \sum_{l=1}^{N} \frac{\xi_{r, l}^{*}}{\left(x+\omega_{r, l}^{*}\right)^{2}}
$$

where $\omega_{r, l}^{*}$ and $\xi_{r, l}^{*}$ are expressed in (64).

Plugging (D.4), (D.5), and (D.6) together yields

$$
\begin{aligned}
\mathrm{OP}_{m, \mathrm{AF}}^{\mathrm{LB}, *}= & 1-\sum_{q=1}^{T_{1}} \sum_{n=1}^{N} \sum_{r=T_{1}+1}^{L_{m}+1} \sum_{l=1}^{N} \int_{0}^{+\infty} \frac{\mu_{1} y}{\left(y+\mu_{2}\right)\left(y+\mu_{3}\right)^{2}} d y \\
= & 1-\sum_{q=1}^{T_{1}} \sum_{n=1}^{N} \sum_{r=T_{1}+1}^{L_{m}+1} \sum_{l=1}^{N} \int_{0}^{+\infty}\left(\frac{\mu_{1} \mu_{2}}{\left(\mu_{2}-\mu_{3}\right)^{2}\left(y+\mu_{3}\right)}\right. \\
& \left.-\frac{\mu_{1} \mu_{2}}{\left(\mu_{2}-\mu_{3}\right)^{2}\left(y+\mu_{2}\right)}-\frac{\mu_{1} \mu_{3}}{\left(\mu_{2}-\mu_{3}\right)\left(y+\mu_{3}\right)^{2}}\right) d y,
\end{aligned}
$$

where $\mu_{1}, \mu_{2}$, and $\mu_{3}$ are given by (64), and $\mu_{2} \neq \mu_{3}$.

After calculating the integrals in (D.7) and substituting the obtained result into (D.2), we obtain (63).

\section{Data Availability}

The simulation data used to support the findings of this study are included within the article.

\section{Conflicts of Interest}

The authors declare that they have no conflicts of interest.

\section{References}

[1] M. O. Hasna and M.-S. Alouini, "Outage probability of multihop transmission over Nakagami fading channels," IEEE Communications Letters, vol. 7, no. 5, pp. 216-218, 2003.

[2] G. Farhadi and N. C. Beaulieu, "On the outage and error probability of amplify-and-forward multi-hop diversity transmission systems," in 2008 IEEE International Conference on Communications, pp. 3748-3754, Beijing, China, 2008.

[3] G. Farhadi and N. C. Beaulieu, "On the performance of amplify-and-forward cooperative systems with fixed gain relays," IEEE Transactions on Communications, vol. 7, no. 5, pp. 1851-1856, 2008.

[4] O. Chughtai, N. Badruddin, M. Rehan, and A. Khan, "Congestion detection and alleviation in multihop wireless sensor networks," Wireless Communications and Mobile Computing, vol. 2017, Article ID 9243019, 13 pages, 2017.

[5] Z. Li and P. Xin, "Evidence-efficient multihop clustering routing scheme for large-scale wireless sensor networks," Wireless Communications and Mobile Computing, vol. 2017, Article ID 1914956, 14 pages, 2017.

[6] C. K. Datsikas, N. C. Sagias, F. I. Lazarakis, and G. S. Tombras, "Outage analysis of decode-and-forward relaying over Nakagami- $m$ fading channels," IEEE Signal Processing Letters, vol. 15, pp. 41-44, 2008. 
[7] A. Behnad, X. Gao, and X. Wang, "Distributed resource allocation for multihop decode-and-forward relay systems," IEEE Transactions on Vehicular Technology, vol. 64, no. 10, pp. 4821-4826, 2015.

[8] J. Yao, S. Feng, X. Zhou, and Y. Liu, "Secure routing in multihop wireless ad-hoc networks with decode-and-forward relaying," IEEE Transactions on Communications, vol. 64, no. 2, pp. 753-764, 2016.

[9] A. Behnad, N. C. Beaulieu, and B. Maham, "Multi-hop amplify-and-forward relaying on Nakagami-0.5 fading channels," IEEE Wireless Communications Letters, vol. 1, no. 3, pp. 173-176, 2012.

[10] A. Behnad and X. Wang, "Accuracy of harmonic mean approximation in performance analysis of multihop amplifyand-forward relaying," IEEE Wireless Communications Letters, vol. 3, no. 2, pp. 125-128, 2014.

[11] K. P. Peppas, P. T. Mathiopoulos, and J. Yang, "On the effective capacity of amplify-and-forward multihop transmission over arbitrary and correlated fading channels," IEEE Wireless Communications Letters, vol. 5, no. 3, pp. 248-251, 2016.

[12] S. S. Ikki and S. Aissa, "Multihop wireless relaying systems in the presence of cochannel interferences: performance analysis and design optimization," IEEE Transactions on Vehicular Technology, vol. 61, no. 2, pp. 566-573, 2012.

[13] T. Soithong, V. A. Aalo, G. P. Efthymoglou, and C. Chayawan, "Outage analysis of multihop relay systems in interferencelimited Nakagami- $m$ fading channels," IEEE Transactions on Vehicular Technology, vol. 61, no. 3, pp. 1451-1457, 2012.

[14] V. A. Aalo, K. P. Peppas, G. P. Efthymoglou, M. M. Alwakeel, and S. S. Alwakeel, "Serial amplify-and-forward relay transmission systems in Nakagami- $m$ fading channels with a Poisson interference field," IEEE Transactions on Vehicular Technology, vol. 63, no. 5, pp. 2183-2196, 2014.

[15] A. A. AbdelNabi, F. S. al-Qahtani, M. Shaqfeh, S. S. Ikki, and H. M. Alnuweiri, "Performance analysis of mimo multi-hop system with TAS/MRC in Poisson field of interferers," IEEE Transactions on Communications, vol. 64, no. 2, pp. 525-540, 2016.

[16] J. Qi, S. Aïssa, and M.-S. Alouini, "Multi-hop amplify-andforward relaying cooperation in the presence of $\mathrm{i} / \mathrm{q}$ imbalance," in 2013 IEEE International Conference on Communications (ICC), pp. 4978-4982, Budapest, Hungary, June 2013.

[17] T. T. Duy, C. N. Trang, V. N. Q. Bao, and T. Hanh, "Joint impact of hardware impairment and co-channel interference on multi-hop relaying," in 2015 International Conference on Advanced Technologies for Communications (ATC), pp. 8892, Ho Chi Minh City, Vietnam, October 2015.

[18] G. Farhadi and N. Beaulieu, "Fixed relaying versus selective relaying in multi-hop diversity transmission systems," IEEE Transactions on Communications, vol. 58, no. 3, pp. 956-965, 2010.

[19] C. Conne, M. Ju, Z. Yi, H.-K. Song, and I.-M. Kim, "Ser analysis and pdf derivation for multi-hop amplify-and-forward relay systems," IEEE Transactions on Communications, vol. 58, no. 8, pp. 2413-2424, 2010.

[20] S. Sharma, Y. Shi, Y. T. Hou, H. D. Sherali, S. Kompella, and S. F. Midkiff, "Joint flow routing and relay node assignment in cooperative multi-hop networks," IEEE Journal on Selected Areas in Communications, vol. 30, no. 2, pp. 254-262, 2012.

[21] U. R. Afonseca, C. F. Rego, and J. L. Bordim, "Carcc: Connectivity autorecovering via cooperative communication," Wire- less Communications and Mobile Computing, vol. 2017, Article ID 4195908, 14 pages, 2017.

[22] Q. Deng and A. G. Klein, "Diversity of multi-hop cluster-based routing with arbitrary relay selection," IET Communications, vol. 6, no. 9, pp. 1054-1060, 2012.

[23] V. N. Q. Bao, T. T. Duy, and N. T. Van, "Exact outage analysis of energy-harvesting multihop cluster-based networks with multiple power beacons over Nakagami-m fading channels," in 2018 2nd International Conference on Recent Advances in Signal Processing, Telecommunications \& Computing (SigTelCom), pp. 1-6, Ho Chi Minh City, Vietnam, January 2018.

[24] M. R. Bhatnagar, "Performance analysis of a path selection scheme in multi-hop decode-and-forward protocol," IEEE Communications Letters, vol. 16, no. 12, pp. 1980-1983, 2012.

[25] M. R. Bhatnagar, R. K. Mallik, and O. Tirkkonen, "Performance evaluation of best-path selection in a multihop decode-and-forward cooperative system," IEEE Transactions on Vehicular Technology, vol. 65, no. 4, pp. 2722-2728, 2016.

[26] T. D. Hieu, T. T. Duy, and B.-S. Kim, "Performance enhancement for multihop harvest-to-transmit wsns with pathselection methods in presence of eavesdroppers and hardware noises," IEEE Sensors Journal, vol. 18, no. 12, pp. 5173-5186, 2018.

[27] J. Mitola and G. Q. Maguire, "Cognitive radio: making software radios more personal," IEEE Personal Communications, vol. 6, no. 4, pp. 13-18, 1999.

[28] J.-P. Hong, B. Hong, T. W. Ban, and W. Choi, "On the cooperative diversity gain in underlay cognitive radio systems," IEEE Transactions on Communications, vol. 60, no. 1, pp. 209-219, 2012.

[29] M. el Tanab, W. Hamouda, and Y. Fahmy, "Distributed opportunistic scheduling for mimo underlay cognitive radio networks," Wireless Communications and Mobile Computing, vol. 16, no. 15, pp. 2212-2224, 2016.

[30] K. Zheng, X.-Y. Liu, X. Liu, and Y. Zhu, "Hybrid overlayunderlay cognitive radio networks with energy harvesting," IEEE Transactions on Communications, vol. 67, no. 7 , pp. 4669-4682, 2019.

[31] H. Phan, H.-J. Zepernick, and H. Tran, "Impact of interference power constraint on multi-hop cognitive amplify-and-forward relay networks over Nakagami-m fading," IET Communications, vol. 7, no. 9, pp. 860-866, 2013.

[32] C. Xu, M. Zheng, W. Liang, H. Yu, and Y.-C. Liang, "Outage performance of underlay multihop cognitive relay networks with energy harvesting," IEEE Communications Letters, vol. 20, no. 6, pp. 1148-1151, 2016.

[33] C. Xu, M. Zheng, W. Liang, H. Yu, and Y.-C. Liang, "End-toend throughput maximization for underlay multi-hop cognitive radio networks with rf energy harvesting," IEEE Transactions on Wireless Communications, vol. 16, no. 6, pp. 35613572, 2017.

[34] H. K. Boddapati, M. R. Bhatnagar, and S. Prakriya, "Performance analysis of cluster-based multi-hop underlay crns using max-link-selection protocol," IEEE Transactions on Cognitive Communications and Networking, vol. 4, no. 1, pp. 15-29, 2018.

[35] H. K. Boddapati, M. R. Bhatnagar, and S. Prakriya, "Performance of incremental relaying protocols for cooperative multihop crns," IEEE Transactions on Vehicular Technology, vol. 67 , no. 7, pp. 6006-6022, 2018. 
[36] T. D. Hieu, T. T. Duy, and S. G. Choi, "Performance enhancement for harvest-to-transmit cognitive multi-hop networks with best path selection method under presence of eavesdropper," in 2018 20th International Conference on Advanced Communication Technology (ICACT), pp. 323328, Chuncheon-si Gangwon-do, Korea (South), February 2018.

[37] L. Lv, J. Chen, Q. Ni, Z. Ding, and H. Jiang, "Cognitive nonorthogonal multiple access with cooperative relaying: a new wireless frontier for $5 \mathrm{~g}$ spectrum sharing," IEEE Communications Magazine, vol. 56, no. 4, pp. 188-195, 2018.

[38] Z. Zhang, G. Yang, Z. Ma, M. Xiao, Z. Ding, and P. Fan, "Heterogeneous ultradense networks with NOMA: system architecture, coordination framework, and performance evaluation," IEEE Vehicular Technology Magazine, vol. 13, no. 2, pp. 110-120, 2018.

[39] M. Aldababsa, M. Toka, S. Gökçeli, G. K. Kurt, and O. Kucur, "A tutorial on nonorthogonal multiple access for $5 \mathrm{~g}$ and beyond," Wireless Communications and Mobile Computing, vol. 2018, Article ID 9713450, 24 pages, 2018.

[40] X. Yue, Y. Liu, S. Kang, A. Nallanathan, and Z. Ding, "Exploiting full/half-duplex user relaying in NOMA systems," IEEE Transactions on Communications, vol. 66, no. 2, pp. 560-575, 2018.

[41] K. Janghel and S. Prakriya, "Performance of adaptive oma/cooperative-NOMA scheme with user selection," IEEE Communications Letters, vol. 22, no. 10, pp. 2092-2095, 2018.

[42] M. A. Sedaghat and R. R. Muller, "On user pairing in uplink NOMA," IEEE Transactions on Wireless Communications, vol. 17, no. 5, pp. 3474-3486, 2018.

[43] F. Kara and H. Kaya, "Ber performances of downlink and uplink NOMA in the presence of sic errors over fading channels," IET Communications, vol. 12, no. 15, pp. 1834-1844, 2018.

[44] G. Im and J. H. Lee, "Outage probability for cooperative NOMA systems with imperfect sic in cognitive radio networks," IEEE Communications Letters, vol. 23, no. 4, pp. 692-695, 2019.

[45] S. Arzykulov, G. Nauryzbayev, T. A. Tsiftsis, and B. Maham, "Performance analysis of underlay cognitive radio nonorthogonal multiple access networks," IEEE Transactions on Vehicular Technology, vol. 68, no. 9, pp. 9318-9322, 2019.

[46] G. Nauryzbayev, S. Arzykulov, T. A. Tsiftsis, and M. Abdallah, "Performance of cooperative underlay CR-NOMA networks over Nakagami-m channels," in 2018 IEEE International Conference on Communications Workshops (ICC Workshops), pp. 1-6, Kansas City, MO, USA, May 2018.

[47] V. N. Vo, C. So-in, H. Tran, and H. Tran, "Optimal system performance in multihop energy harvesting WSNS using cooperative NOMA and friendly jammers," IEEE Access, vol. 7, pp. 125494-125510, 2019.

[48] N. Pham Thi Dan, D. Tran Trung, K. Ho-van, and N. Thanh Binh, "Performance evaluation of best path selection protocol in multi-hop relaying networks under joint impact of co-channel interference and hardware impairments," Journal of Science and Technology: Issue on Information and Communications Technology, vol. 4, no. 1, pp. 3338, 2018.
[49] E. Bjornson, M. Matthaiou, and M. Debbah, "A new look at dual-hop relaying: performance limits with hardware impairments," IEEE Transactions on Communications, vol. 61, no. 11, pp. 4512-4525, 2013.

[50] E. Bjornson, J. Hoydis, M. Kountouris, and M. Debbah, "Massive MIMO systems with non-ideal hardware: energy efficiency, estimation, and capacity limits," IEEE Transactions on Information Theory, vol. 60, no. 11, pp. 7112-7139, 2014. 